\title{
Kinase domain-targeted isolation of defense-related receptor-like kinases (RLK/Pelle) in Platanus $\times$ acerifolia: phylogenetic and structural analysis
}

Massimo Pilotti ${ }^{1 *}$, Angela Brunetti ${ }^{1}$, Paolo Uva ${ }^{2}$, Valentina Lumia ${ }^{1}$, Lorenza Tizzani ${ }^{1}$, Fabio Gervasi ${ }^{3}$, Michele lacono ${ }^{4}$ and Massimo Pindo ${ }^{5}$

\begin{abstract}
Background: Plant receptor-like kinase (RLK/Pelle) family regulates growth and developmental processes and interaction with pathogens and symbionts.

Platanaceae is one of the earliest branches of Eudicots temporally located before the split which gave rise to Rosids and Asterids. Thus investigations into the RLK family in Platanus can provide information on the evolution of this gene family in the land plants.

Moreover RLKs are good candidates for finding genes that are able to confer resistance to Platanus pathogens.

Results: Degenerate oligonucleotide primers targeting the kinase domain of stress-related RLKs were used to isolate for the first time 111 RLK gene fragments in Platanus $\times$ acerifolia. Sequences were classified as candidates of the following subfamilies: CrRLK1L, LRR XII, WAK-like, and LRR X-BRI1 group. All the structural features typical of the RLK kinase domain were identified, including the non-RD motif which marks potential pathogen recognition receptors (PRRs). The LRR XII candidates, whose counterpart in Arabidopsis and rice comprises non-RD PRRs, were mostly non-RD kinases, suggesting a group of PRRs. Region-specific signatures of a relaxed purifying selection in the LRR XII candidates were also found, which is novel for plant RLK kinase domain and further supports the role of LRR XII candidates as PRRs. As we obtained CrRLK1L candidates using primers designed on Pto of tomato, we analysed the phylogenetic relationship between CrRLK1L and Pto-like of plant species. We thus classified all non-solanaceous Pto-like genes as CrRLK1L and highlighted for the first time the close phylogenetic vicinity between CrRLK1L and Pto group. The origins of Pto from CrRLK1L is proposed as an evolutionary mechanism.
\end{abstract}

Conclusions: The signatures of relaxed purifying selection highlight that a group of RLKs might have been involved in the expression of phenotypic plasticity and is thus a good candidate for investigations into pathogen resistance.

Search of Pto-like genes in Platanus highlighted the close relationship between CrRLK1L and Pto group. It will be exciting to verify if sensu strictu Pto are present in taxonomic groups other than Solanaceae, in order to further clarify the evolutionary link with CrRLK1L.

We obtained a first valuable resource useful for an in-depth study on stress perception systems.

Keywords: Platanus $\times$ acerifolia, RLK/Pelle, Pto-like, CrRLK1L, LRR XII, WAK-like, LRR X-BRI1, Relaxed purifying selection, Pathogen resistance

\footnotetext{
* Correspondence: massimo.pilotti@entecra.it

${ }^{1}$ Plant Pathology Research Center, CRA-PAV Agricultural Research Council,

V. C.G. Bertero 22, 00156 Rome, Italy

Full list of author information is available at the end of the article
} 


\section{Background}

In animals, cell surface receptor tyrosine kinases (RTKs) and serine/threonine kinase receptors (STRKs) are of primary importance for the perception of intercellular signals and the transduction of signals at an intracellular level. In general the domain composition of these receptors is defined by an extracellular ligand-binding domain, a single-pass trans-membrane domain and a cytoplasmic tyrosine or serine/threonine kinase domain [1,2]. In the plant lineage, the counterpart of RTKs and STRKs is represented by the family of receptor-like kinases (RLKs), which share the same domain composition [3]. The RLK kinase domain, a serine-threonine kinase, belongs to the same gene family as the Drosophila melanogaster Pelle and mammalian interleukin receptor-associated kinases. Thus the term RLK/Pelle was established [3,4].

With regard to RLK/Pelles, a striking difference between the land plant and non-plant eukaryote genomes is the size of this family, in fact 329 genes have been identified in moss (Physcomitrella patens), 610 in Arabidopsis, 647 in tomato, 1192 in poplar (Populus trichocarpa), 1070 in rice, 1 and 4 in the animals Drosophila melanogaster and Homo sapiens respectively, and none have been identified in fungal organisms [5,6]. It has been suggested that one of the evolutionary reasons for the gene family's expansion derives from the need to cope, as sessile organisms, with an ever-changing environment, including a multitude of mutating pathogenic and would be-pathogenic microbes $[4,5,7]$. This would account for the variety of RLK/ Pelle types - 53 subfamilies categorized in Arabidopsis - for the genetic redundancy present in part of the subfamilies, and for the up-regulation of hundreds of RLK/Pelle members under biotic stress [3-5,7].

Some members within the plant RLK/Pelle family, have been shown to be transmembrane receptors $[8,9]$ and it is likely that this is the main functional contribution of these proteins [4]. Two functional groups are recognized in plants: RLKs controlling growth and development processes, and RLKs regulating interactions with microbes, both pathogens and symbionts. The rice (Oryza sativa) $\mathrm{Xa} 21$ is one example of the latter class, which confers resistance to the bacterium Xanthomonas oryzae pv. oryzae [10]. Other examples include EFR (EF-TU RECEPTOR) of Arabidopsis thaliana which is a PAMP (Pathogen Associated Molecular Pattern) receptor [11], and Arabidopsis RFO1 (RESISTANCE TO FUSARIUM OXISPORUM 1) which confers broad spectrum resistance to diverse Fusarium oxisporum races and Verticillium longisporum $[12,13]$.

A functional dualism may involve BRI1 (BRASSINOS TEROID-INSENSITIVE1). This RLK/Pelle has an important role in the regulation of growth and development through the perception of brassinosteroids (BRs) $[14,15]$. Its involvement in stress responses is suggested by the fact that BRs are also strongly related in stress signaling [16].
Furthermore, a tomato BRI1 ortholog, SR160 (SYSTEMIN RECEPTOR 160), is able to bind systemin, a solanaceous specific protein which induces systemic wound responses following attack by feeding insects [17] although it does not represent the functional systemin receptor $[18,19]$.

A lack of extracellular domain is a characteristic of another class of the plant RLK/Pelle family, the receptorlike cytoplasmic kinases (RLCKs) [4]. Pto (for Pseudomonas syringae pv. tomato) is a well-known member of this class and confers resistance to the bacterial pathogen, $P$. syringae pv tomato in wild tomato species, according to a host genotype/pathogen race specificity fashion [20]. Great interest in Pto orthologs in plant species has arisen from the fact that the overexpression of Pto has been shown to confer a broad spectrum resistance in tomato [21].

Platanaceae is a tree family that is considered as one of the earliest branches of Eudicots [22] (http://www.mobot. org/MOBOT/research/APweb/.). In contrast, Platanus $\times$ acerifolia (Ait.) Willd. is a species of this family which originated in the 17th century from a cross between individuals belonging to the $P$. occidentalis $\mathrm{L}$. species group and $P$. orientalis L. [23,24]. Therefore, $P . \times$ acerifolia (Pac) combines genomic contributions from the two parental species, which evolved separately for long geological periods [24]. The high haploid chromosome number, $\mathrm{n}=21$, suggests that the current Platanaceae had ancient polyploid origins [25] which adds to the overall complexity. Given these features, investigations into the RLK/Pelle family in Pac can provide information on the nature of this gene family in the Dicots, especially when compared with the Arabidopsis counterpart.

Platanaceae suffer from several diseases including canker stain, a vascular disease caused by the fungus Ceratocystis platani (J.M. Walter) Engelbr. \& T. C. Harr., and antrachnose, caused by the fungus Apiognomonia veneta (Sacc. et Speg.), which are particularly destructive and have heavily conditioned the diffusion and cultivation of Platanus spp. [26,27]. Thus unravelling the resistance response of Platanus to pathogens is necessary in order to sustain and speed up current time-consuming genetic selection programmes $[24,28]$. The plant RLK/Pelle family is thus a good candidate for finding genes that are able to confer resistance to pathogens.

The aim of this study was to isolate RLK/Pelle genes in Pac which, based on knowledge of Arabidopsis, play a potential role in pathogen perception and signal transduction. We successfully applied a PCR-based strategy, which targeted a representative region of the kinase domain.

We isolated for the first time RLK/Pelle gene fragments in Pac, with an uninterrupted open reading frame (ORF). Sequence analyses clearly showed that the genes were, in most cases, candidates of the following RLK/Pelle subfamilies: CrRLK1L, LRR XII, WAK-like, LRR X-BRI1 group. A number of structural motifs and the analysis of 
non-synonymous versus synonymous substitution rates, confirmed the phylogenetic identification and provided an insight into their potential functions. In fact, the detection in Pac of signals of a completely relaxed purifying selection in sub-regions of the kinase domain, is another important finding of this study.

As we isolated CrRLK1L-L using primers designed on Pto of tomato, we also wished to clarify the relationships between Pto-like of several plant species available in the GenBank, the bona fide Pto family of solanaceous species and CrRLK1L. As a result a close phylogenetic relationship was revealed between Pto and CrRLK1L.

\section{Methods}

Plant material, nucleic acid extraction and primer design Genomic DNA was isolated from young leaves collected in the spring from a potted Pac tree (accession MS.12) using a Plant Midi Kit (Qiagen, Hilden, Germany) according to the manufacturer's instructions.

Degenerate primers were designed in order to isolate RLK/Pelle candidate genes for pathogen perception and signal transduction: 1) known $\mathrm{R}$ genes (Pto, Xa-21, $X a-26, R F O 1)$; 2) PAMP receptor genes (EFR); 3) the whole BRI1 gene group. The primers were designed within the kinase catalytic domain. They were generated from the nucleotide stretches conserved in the model gene and several plant homologs, available from the NCBI GenBank database. In the case of RFO1, a WAK-like gene, collected sequences were used to infer a phylogenetic tree in order to identify clades of homologous WAK-like genes from which distinct sets of primers were generated. We then used a similar strategy in order to search for BRI1-like genes.

All the information regarding primer is reported in Additional file 1.

\section{Amplification and cloning of gene fragments}

PCRs were performed in a $50-\mu \mathrm{l}$ reaction volume containing each dNTP at $200 \mu \mathrm{M}$, each primer at $1 \mu \mathrm{M}, 2.5 \mathrm{U}$ of Platinum Taq DNA Polymerase High Fidelity (Invitrogen), $2 \mathrm{mM} \mathrm{MgSO}_{4}$, and 100-200 ng of genomic DNA as a template, in the buffer supplied by the manufacturer. The initial denaturation step was carried out at $94^{\circ} \mathrm{C}$ for $2 \mathrm{~min}$, followed by 35 amplification cycles $\left(94^{\circ} \mathrm{C}\right.$ for $1 \mathrm{~min}$, annealing at $47^{\circ} \mathrm{C}$ for $50 \mathrm{~s}$, an extension at $68^{\circ} \mathrm{C}$ for $1 \mathrm{~min}$ ) and a final extension step at $68^{\circ} \mathrm{C}$ for $15 \mathrm{~min}$. Analysis of PCR products, cloning and sequencing was as previously described [29]. A variable number of clones per cloned DNA band were sequenced in both directions (Bio Fab Research) (Additional file 2). RLK/Pelle-like sequences were deposited in the GenBank database [GenBank: EU722764-EU722869, EU722871-EU722900, HQ425329-30] (Additional file 3).

\section{Alignments and phylogenetic analyses}

Clone sequences were trimmed of vector and primer sequences and were then considered for further analysis. To select gene fragments representing the kinase-catalytic region, the amino acid sequences were compared with UniProt using blastp [30]. Multiple sequence alignments were performed with MAFFT [31]. Pairwise local alignments were performed using the EMBOSS Pairwise Alignment suite [32] (gap open: 10; gap extend: 0.5; matrix: Blosum62).

Since we isolated portions of the full length ORF, we classified the Pac sequences according to the nomenclature by Shiu and Bleecker [4] or the name of the gene for which a potential orthology relation was evident, followed by the code $-\mathrm{L}(=-$ like).

Phylogenetic analysis was based on the MAFFT alignment of the amino acid sequences of the kinase-catalytic domain. Trees were inferred using the Neighbor-joining (NJ) method [33], with 1,000 bootstrap replications. The evolutionary distances were computed using the Poisson correction method with rate uniformity among sites [34] and were in the units of the number of amino acid substitutions per site. Analyses were conducted in Mega version 6 [35].

Phylogenetic analyses were also conducted with two maximum-likelihood (ML) methods: i) Whelan And Goldman substitution model - WAG [36], with 1,000 bootstrap replications and rate uniformity among sites (analyses were conducted in Mega version 6). ii) $\mathrm{ML}$ approach implemented in the TREE-PUZZLE software package [37], with the following parameters: VT (variable time) substitution model [38], gamma-distributed rates of heterogeneity among sites, amino acid frequencies inferred from the data set, branch lengths estimated without the molecular-clock assumption, 50,000 puzzling steps to infer branch support values for the relative majority consensus tree.

To classify Pac sequences we made a phylogenetic comparison with representative members of all the subfamilies of the Arabidopsis RLK/Pelle complement [4]. Then sequences were divided into homology groups and each group was compared with the closest whole RLK/Pelle subfamily.

We also performed a comparative phylogenetic analysis in order to shed light on the relations between the Pto group [39], Pto-like of Solanaceae, Pto-like of other dicot and monocot plants, CrRLK1L, and the Pto-primer-derived sequences obtained in this work.

Apart from Arabidopsis, genes from nine additional full genome-sequenced species and partial sequences from a number of plant species were included in the phylogenies for comparison. All the reference sequences and those used to root the trees are reported in Additional file 4.

In the phylogenetic trees, sequence names contain: I) the acronym of the protein or, secondly, the locus tag or 
the accession number, II) the Latin name of the species, and III) the RLK/Pelle subfamily to which the protein belongs. Latin names were abbreviated as follows: Arab. thal. = Arabidopsis thaliana, Caps. = Capsicum, Cath. = Catharantus, Frag. = Fragaria, Mu. = Musa, Nicot. tab. = Nicotiana tabacum, Ory. = Oryza, Phas. $=$ Phaseolus, Pop. $=$ Populus, Pot. = Potentilla, Ric. $=$ Ricinus, Sol.pimp. = Solanum pimpinellifolium, Sol. lyc. $=$ Solanum lycopersicum, Vit. $=$ Vitis, Physc.patens $=$ Physcomitrella patens.

\section{Domain detection and determination of other structural features}

Domain detection was performed using Pfam [40] and SMART [41].

The phylogenetic classification at the subfamily level was validated by comparing some representative Pac sequences with long isotigs generated from a 454 GS-FLX transcriptome data set of the same species, though using a different genotype (Pilotti, Brunetti, Iacono and Pindo, unpublished data) (Additional file 5). Putative extracellular domains of the isotigs were identified as described above.

Serine-threonine and tyrosine protein kinase motifs [42] were searched for and a consensus was determined by using the motif-based sequence analysis tool MEME [43].

We compared the activation segment of Pto and BRI1 with the corresponding region of the deduced protein of the entire Pac RLK/Pelle-like sequence complement and the corresponding RLK/Pelle subfamilies of Arabidopsis.

\section{Non-synonymous versus synonymous substitution rates}

The ratio of non-synonymous (Ka) to synonymous (Ks) nucleotide substitutions was calculated for pairwise comparisons using a 75 bp sliding window with a step size of $15 \mathrm{bp}$. The analysis was based on codon-delimited nucleotide alignments and performed with JCoDA [44] which uses the PAML (yn00) suite [45] and the Yang and Nielsen substitution model [46]. JCoDA was also used to identify the individual sites under positive selection (site by site): the site models M7 and M8, which account for transitional rate bias and unequal codon frequencies, were applied, and the differences were assessed with a likelihood ratio test $($ d.f. $=2)$. The tree topology required by the two models was inferred from the amino acid sequence by the maximum likelihood approach using the Phylip package [47], with a JTT substitution matrix [48] and rate heterogeneity approximated using a discrete $\gamma$-distribution with four categories. The posterior probabilities of individual sites belonging to the class $\mathrm{Ka} / \mathrm{Ks}>1$ were calculated using the Bayes empirical Bayes approach implemented in PAML.

Codon-delimited alignments were also used to map those positions that were subject to non-synonymous variations in order to: i) identify those that were shared among the different phylogenetic LRR XII-L groups of Pac and LRR XII of Arabidopsis, ii) to describe the amino acid variability at each position.

\section{Results}

Amplification pattern and oligonucleotide efficiency

Generally PCRs based on degenerate oligonucleotide pairs always gave a clear amplification pattern with minimum or no smearing. Evident expected-sized DNA bands were always present and a variable number of clones were sequenced for each band. In some cases additional non expected bands of variable intensity were also present but these were not investigated further.

Nucleotide sequences of the cloned amplicons revealed that oligonucleotide pairs worked highly efficiently at priming the amplification of target genes. Amplicons from 16 oligonucleotide pairs-based PCRs, out of 23, yielded RLK/Pelle-like sequences with maximum efficiency (100\%) (Additional file 2). A total of 111 out of 136 RLK/ Pelle candidates showed an uninterrupted ORF. These sequences represent the focus of this paper. Their length is indicated in Table 1. A total of 26 RLK/Pelle candidates showed interrupted ORFs due to frameshift indels - probably pseudogenes or truncated genes - and were not analysed further.

\section{Phylogenetic analyses for RLK/Pelle subfamily identification}

By using reference sequences of full genome-sequenced species, we initially verified that highly/well supported clades did not change between phylogenies based on

\section{Table 1 RLK/Pelles obtained in Pac}

\begin{tabular}{|c|c|c|c|c|c|}
\hline $\begin{array}{l}\text { Homologous RLK/ } \\
\text { Pelle subfamily }\end{array}$ & No & $\begin{array}{l}\text { Subdomain } \\
\text { spanning }\end{array}$ & $\begin{array}{l}\text { Length } \\
\text { (nt) }\end{array}$ & $\begin{array}{l}\text { non-RD } \\
\text { kinases }\end{array}$ & $\begin{array}{l}\text { Atypical } \\
\text { kinases }\end{array}$ \\
\hline \multirow[t]{2}{*}{ CrRLK1L } & 28 & $\mathrm{I} \rightarrow \mathrm{IX}(27)$ & $495-538$ & - & - \\
\hline & & $\| \rightarrow I X(1)$ & & & \\
\hline \multirow[t]{2}{*}{ LRR-XII } & 34 & $I \rightarrow$ VIII (33) & $442-508$ & 31 & 2 \\
\hline & & $\mathrm{I} \rightarrow \mathrm{VII} / \mathrm{VIII}(1)$ & & & \\
\hline \multirow[t]{3}{*}{ WAK-like } & 28 & $\mathrm{I} \rightarrow \mathrm{VIII/IX(7)}$ & $503-780$ & 1 & 1 \\
\hline & & $\mathrm{I} \rightarrow \mathrm{IX}(17)$ & & & \\
\hline & & $I \rightarrow X I(4)$ & & & \\
\hline \multirow[t]{2}{*}{ LRR X-BRI1 group } & 7 & $I \rightarrow I X / X(4)$ & $628-876$ & - & - \\
\hline & & $\mathrm{I} \rightarrow \mathrm{XI}$ (3) & & & \\
\hline Uncertain: RKF3 & 3 & $I \rightarrow V I I I / I X$ & $549-582$ & - & - \\
\hline L-Lectin & 4 & $I \rightarrow V I I I$ & 494 & - & - \\
\hline LRR-VII & 2 & $I \rightarrow V I I I$ & 460 & 2 & 2 \\
\hline SD-2 & 2 & $\mathrm{I} \rightarrow \mathrm{VIII/IX}$ & 490,492 & 2 & - \\
\hline Uncertain: & 3 & $\mathrm{I} / \mathrm{I} \rightarrow \mathrm{VIII}$ & 448 & 3 & - \\
\hline \multicolumn{6}{|l|}{ Thaumatin/LRK10L-2/ } \\
\hline GDPD/CRPK1-like-2 & & & & & \\
\hline
\end{tabular}


the partial kinase domain and those inferred with the complete kinase domain or the full ORF. A comparison with CrRLK1L and WAK-like phylogenies in other studies $[49,50]$ further confirmed the robustness of our methodology (data not shown).

A highly supported clustering showed that close homologs of CrRLK1L, LRR XII, WAK-like and LRR X-BRI1 group, had been isolated (Additional file 6A and $\mathrm{B}$ ).

CrRLK1L candidates (CrRLK1L-L) were obtained with Pto primers. All clustered with a maximum bootstrap value with CrRLK1L of Arabidopsis and rice (Figure 1, Additional file 6 and Additional file 7) [50]. Specifically these candidates fell into the largest group of CrRLK1L, previously defined as CRPK1-like1 [4]. In the highlysupported CRPK1-like1 clade, Pto and its relatives of $L$. pimpinellifolium were also included, and clustered together with a maximum bootstrap value. The specific Pto clade did not include any CrRLK1L. The unknown sequences of poplar, which were used for comparison, were all included in the highly/well-supported subgroups headed by Pac sequences and the CrRLK1L reference sequences (Figure 1, Additional file 6 and Additional file 7). Using SMART and Pfam predictive tools, we identified the poplar sequences as CrRLK1L (see "Re-classification of plant Pto-like genes").

With regard to the sequences obtained with Xa21/Xa26/ $E F R$ primers, phylogenetic analyses clearly showed that they were LRR XII candidates (LRR XII-L) (Additional file 6, Figure 2). Founding members of LRR XII are FLS2 and EFR in Arabidopsis and Xa21 in rice [7,11]. In general, homologs of the different species did not strictly group at low hierarchical levels (Figure 2). This characteristic of the LRR XII subfamily has been previously shown in genome wide analyses [7].

The use of $\mathrm{Xa21/Xa26/EFR}$ primers also yielded a few sequences which were homologous with other RLK/Pelle subfamilies (Additional file 6 and Additional file 8).

The phylogenetic analyses of sequences obtained with WAK primers clearly identified most of these sequences as WAK-like-L (Additional file 6, Figure 3). The topology of the tree depicted in Figure 3 suggested that the gene complement isolated in $\mathrm{Pac}$ was at least partially representative of the Arabidopsis subfamily structure. In fact, sequences of the two species clustered within four highly supported groups, of which three were species-specific. On the other hand, the group marked by WAKL14 and WAKL21 of Arabidopsis and LeWAK of tomato [51], included members from different species (included $\mathrm{Pac}$ ) with a high statistical support (Figure 3). Multiple alignment of the kinase domain portion spanned by Pac sequences, enabled us to identify four conserved residues, which in the Arabidopsis WAK-like were exclusive to the WAK1 clade: Ile-427, Ser-501, Ser-502, Ile-531 (numbering refers to WAK1). Of the Pac sequences, only those of clade II shared all these residues (Additional file 9). Of the sequences obtained with WAK primers (thirty-one), three showed a homology with RFK3 (RECEPTOR-LIKE KINASE IN FLOWERS 3, subfamily RFK3-Like) of Arabidopsis and were not analysed further.

Regarding the sequences obtained with BRI1(-like) primers, phylogenies clearly classified the Pac sequences as BRI1 group candidates (LRR X-BRI1-L) (Additional file 6, Figure 4). In fact sequences strictly clustered in the three highly/well supported sub-clades headed by BRI1, BRL1/BRL3 and BRL2 of Arabidopsis, together with poplar and rice orthologs. The percentage of sequence identity, similarity and the number of gaps of these sequences compared to Arabidopsis BRI1(-like) proteins agree with the phylogeny results (Figure 4).

All ML analyses - Mega 6 and TREE-PUZZLE implemented - confirmed results of the NJ analyses (Additional file 10).

To further confirm the reliability of the phylogenetic classification at the subfamily level, we compared selected sequences of $\mathrm{Pac}$, for each homology group, with ten isotigs generated from a 454 transcriptome dataset derived from $P . \times$ acerifolia. Nearly all the isotigs spanned the whole region represented by PCR RLK/Pelle fragments and matched them with a high/maximum nucleotide identity score. In the isotigs all the putative extracellular domains typical of CrRLK1L, LRR XII, WAK-Like and LRR X were detected thus confirming the phylogenetic identification (Additional file 5).

\section{Re-classification of plant Pto-like genes}

The apparent failure to collect potential orthologs of Pto gene family members, prompted us to investigate the existence of sensu strictu Pto orthologs (or close homologs) in plant species using NJ and ML phylogenetic analyses. We used two approaches.

In the first approach we compared Pto locus and Pto-like of solanaceous species with Pto-like partial sequences of non-solanaceous species (including Pac) and the best Pto blastp matches of some fully sequenced non-solanaceous species. As F. vesca and P.vulgaris genomes have been also fully sequenced, we also incuded the best matches obtained from these genomes using Pto-like partial sequences of $P$. vulgaris and cultivated/wild strawberries as query. Representative CrRLK1L were included as control. All these sequences, clustered together with a high support (Figure 5; Additional file 6A). Within this clade, the branching pattern determined several highly/well supported groups among which one included the Pto locus and the vast majority of Pto-like sequences of Solanaceae. The other clades were headed by CrRLK1L sequences and contained all Pto-like partial sequences of non-solanaceous species, the best Pto and Pto-like blastp matches of fully 


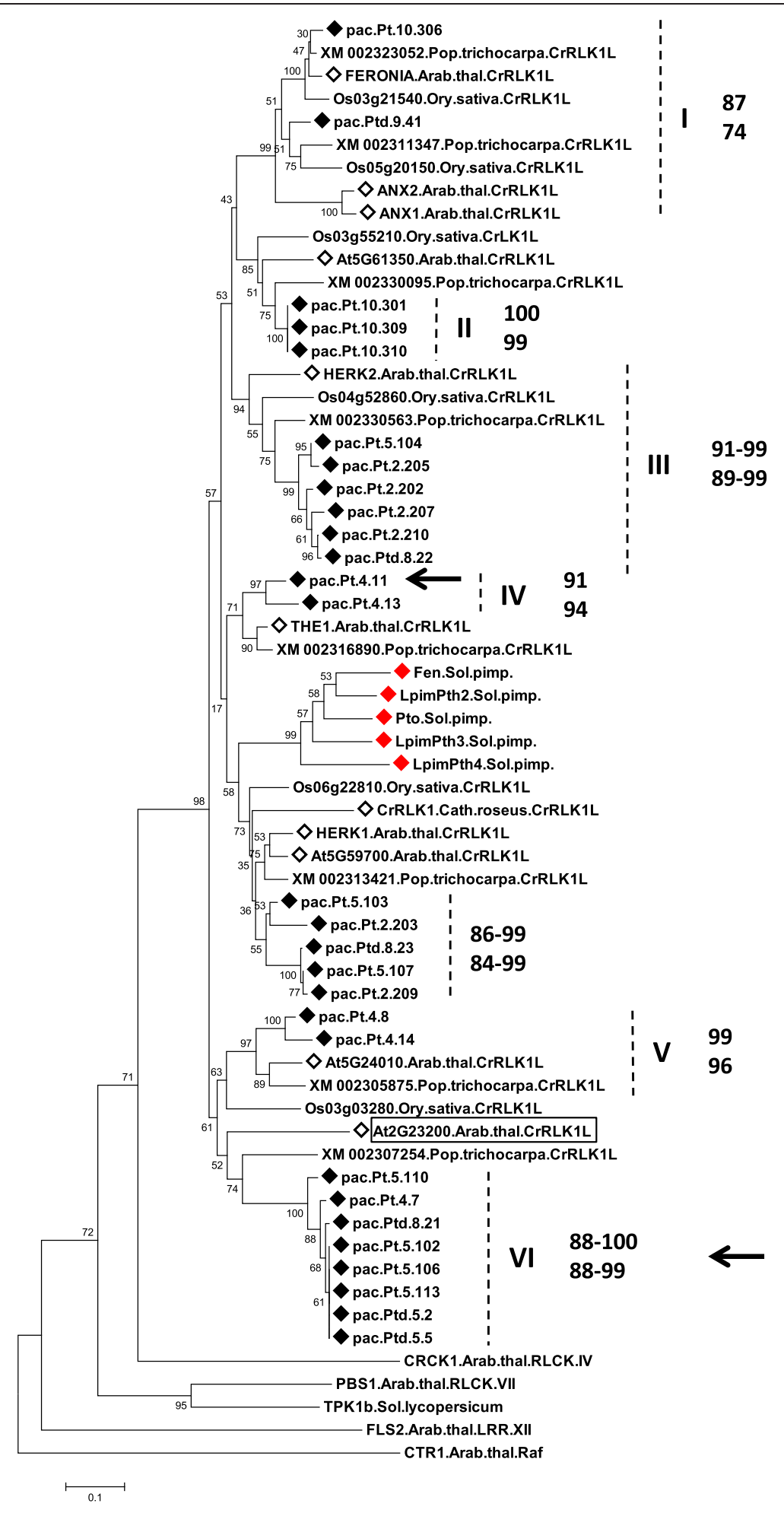

Figure 1 (See legend on next page.) 
(See figure on previous page.)

Figure 1 Neighbor-joining tree of the aa sequences of Pac obtained with Pto primers. Pac sequences: pac, As a comparison we included representative CrRLK1L sequences of Arabidopsis and Catharanthus roseus ( $)$ ), poplar and rice, Pto and paralogs of Solanum piminellifolium (red colored rhombus). Black arrows indicate Pac sequences sharing Thr204 of Pto, which is the main structural determinant of Pto for the specific recognition of AvrPto [59,62]. The rectangular frame indicates the Arabidopsis CrRLK1L member that directly binds to the effector AvrPto of Pseudomonas syringae pv tomato [63]. The kinase domain region spanned subdomain I through IX. The percentage of replicate trees in which sequences clustered together in the bootstrap test (1000 replicates) are shown for each node. The tree is drawn to scale with branch length in the units of the number of amino acid substitution per site (scale at the bottom). Roman numerals indicate groups with a high bootstrap. Arabic numerals indicate amino acid (upper) and nucleotide (lower) identity scores in Pac sequences within each group. Abbreviations are explained in the text.

sequenced non-solanaceous species and a few sequences of Solanaceae (Figure 5). For the sake of legibility, the phylogenetic tree in Figure 5 contains representative sequences of those listed in Additional file 4, which were all tested in a preliminary analysis with the same results. The best Pto blastp matches of Arabidopsis and rice corresponded to CrRLK1L members. Those of poplar, grapevine and castor bean and the best matches from F. vesca and P. vulgaris, obtained using non solanaceous Pto-like as query, were unknown genes with a C-terminal kinase domain, and 1 or $2 \mathrm{~N}$-terminal malectin domains (Di-glucose binding within endoplasmic reticulum). Malectin is a membraneanchored endoplasmic reticulum protein which is highly conserved in animals and recognizes glucose oligomers [52]. The same domain composition was detected in the Arabidopsis CrRLK1L, confirming the identity of poplar, grapevine, castor bean, bean and strawberry proteins as CrRLK1L. Results of NJ analysis were confirmed by the ML pylogenetic analyses - Mega6 and TREE-PUZZLE implemented (Additional file 11).

In the second approach we compared the complete CrRLK1L subfamily of those species in which this gene set had been characterised (i.e. arabidopsis, poplar, tomato, and rice) with Pto locus (see Additional file 4 for sequence sources). Moss (Physcomitrella patens) was also included in the analysis as a representative of basal lineages of land plant. CrRLK1L sequences of moss were retrieved from NCBI GenBank using CrRLK1L of Arabidopsis and Pto as query. The vast majority of the sequences grouped together in highly supported multi-species sub-clades confirming that in CrRLK1L, orthologs are more similar than paralogs. On the contrary Pto and paralogs and the other ortologs from tomato database formed a highly supported Solanaceae-specific clade. The phylogenetic analysis was performed with NJ and two ML methods, which gave the same results (Additional file 12).

Representative non-solanaceous Pto-like partial sequences were each compared with close CrRLK1L relatives and with Pto. Identities and similarity values were always clearly higher with the CrRLK1L relatives than with Pto. In line with this, the number of gaps was lower in CrRLK1L than with Pto. The same analysis, performed on representative Pac CrRLK1L-L, gave similar results (Table 2).

\section{Structural features of the kinase domain: typical, atypical,} $\mathrm{RD}$ and non-RD kinases

The RLK/Pelle-like fragments of Pac spanned subdomain I to subdomain VIII-XI (Table 1). Invariant residues of kinases were all conserved except in five sequences (belonging to LRR XII-L, WAK-like-L and LRR VII-L), which were therefore classified as atypical kinases [53] (for details see Additional file 13).

Based on the crucial role of the RD motif in the catalysis [54], we assessed the nature of Pac sequences as RD or non-RD kinases, by verifying the presence/absence of the conserved Arg (R) immediately preceding the invariant catalytic Asp (D) in subdomain VIB of the catalytic domain (Additional file 13). Most possessed the RD-Arg. Thirty-nine sequences out of 111 (35\%) showed the feature of non-RD kinases. Of the 34 LRR XII-L sequences, 31 were non-RD kinases, and the vast majority (29) had a Cys in substitution of Arg (Figure 2). A single WAK-like-L sequence was also a non-RD kinase (Figure 3). Seven additional non-RD kinases were found among the RLK-like belonging to the unwanted subfamilies (Additional file 8). Features of the non-RD motif of $P a c$ sequences were strongly conserved in the reference sequences of the other plant species, monocot and dicot, with which they grouped (Figure 2, Additional file 8). Specifically Arabidopsis LRR XII were nearly all non-RD, with a Cys substituting Arg.

In the CrRLK1L(-L), WAK-like(-L) and LRR XII(-L) groups of Pac and Arabidopsis, the MEME-based consensus motifs were clearly different from the tyrosine motif and instead there was a clear vicinity with the serine/ threonine or the dual specifity motif (Additional file 14).

\section{Analysis of the activation segment}

In protein kinases, the activation segment is the catalytic region and is made up of two recognizable regions: the T-loop in which regulatory autophosphorylation often occurs and the $\mathrm{P}+1$ loop, which forms the primary binding site for the substrate [55]. The function of several residues, in the activation segment of Pto and BRI1, has been clarified [56,57]. Our objective was to compare the activation segment of Pto and BRI1 with the corresponding regions in the Pac and Arabidopsis CrRLK1L(-L), WAK-like(-L) and BRI1(-L). The LRR XII(-L) sequences were not analyzed as they did not span the entire region of the activation segment. 


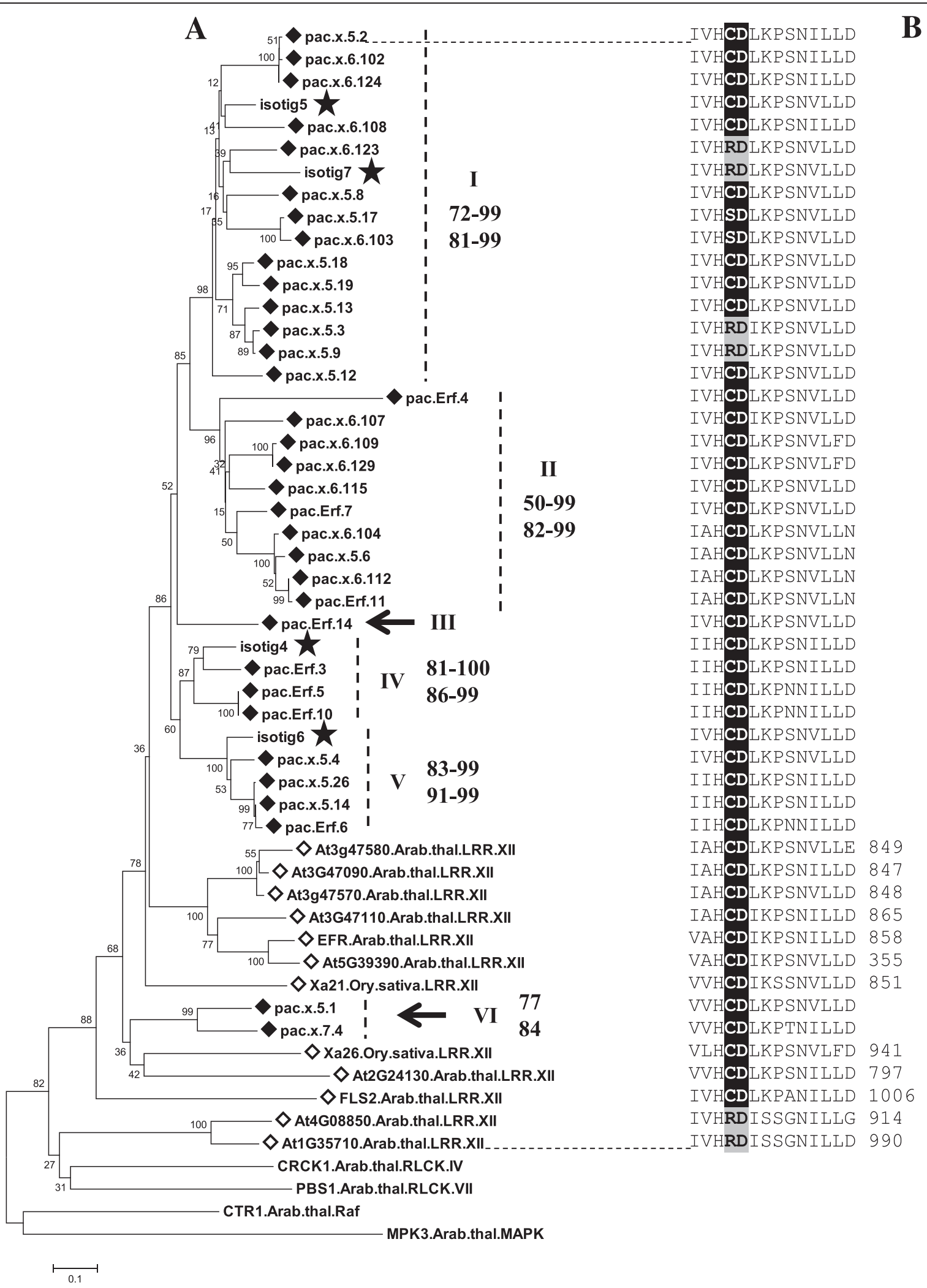

Figure 2 (See legend on next page.) 
(See figure on previous page.)

Figure 2 LRR XII-L of Pac compared with Arabidopsis RLK/Pelle subfamily LRR XII. A Neighbor-joining tree of the aa sequences of LRR XII-L of Pac (pac, $)$ ), the complete set of LRR XII of Arabidopsis $(\diamond)$, two well-known members of LRR XII of rice - Xa21 and Xa26 $(\diamond)$, and isotigs extracted from a 454 transcriptome dataset of Pac which have LRR signatures located upstream the kinase domain (black star) (Additional file 5). Analysis was based on the kinase domain region spanning subdomain I/II up to the beginning of VIII. See caption of Figure 1 for additional details on the representation of the tree. $\mathbf{B}$ Multiple alignment of the RD motif (shaded grey) and non-RD motif (shaded black) corresponding to the sequences included in the tree.

We identified a motif spanning the activation segment

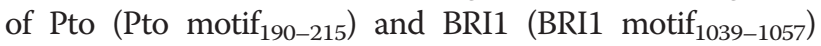
and composed of residues that are crucial for their function (Figure 6, Additional file 15), [56-64]. When the two motifs were aligned, six out of seven key residues of the BRI1 motif coincided with key residues of the Pto motif 190-215 $_{15}$ at the corresponding positions (Figure 6, Additional file 15). Specifically alignment of BRI1 Thr-1045 was ambiguous. As this residue is involved in phosphorilation and is coupled to a serine (Ser-1044) similarly to Pto Thr-199, we decided to locate the two threonines in corresponding positions by re-locating the gaps as needed (Figure 6, Additional file 15). As a consequence we choose to consider BRI1 Ser-1044 an insertion, with BRI1 Ser-1042 and Pto Ser-198 located in corresponding positions (Figure 6, Additional file 15). Alternatively BRI1 Ser-1042 may be considered an insertion with Pto Ser-198 and BRI1 Ser-1044 located in corresponding positions (data not shown).

A search for the motifs in the above cited sequences, highlighted that many or all residues recurred in the sequence complements (Figure 6, Additional files 16, 17 and 18). The motifs were also partially conserved in BAK1, ERECTA and FLS2, which were included in the analysis for comparison (Figure 6).

Given the results of the phylogenetic reconstruction and the analyses of structural features (RD, non-RD, typical, atypical motifs and the activation segment), we defined the RLK/Pelle-like of Pac as bona fide RLK/Pelles.

\section{Non-synonymous versus synonymous substitution rates, non-synonymous positions and their amino acid variability}

In a preliminary work we calculated $\mathrm{Ka} / \mathrm{Ks}$ values in sequence pairs within CrRLK1L(-L), WAK-like(-L) and LRR XII(-L) groups, for Pac and Arabidopsis separately. The analysis revealed clear signatures of purifying selection in both species. Interestingly in $\mathrm{Pac}$, the distribution of $\mathrm{Ka} /$ Ks values of LRR XII-L was shifted in higher values - in any case less than 1 - compared with Arabidopsis. A significant increase was also evident in the LRR XII(-L) compared with WAK-Like(-L) and CrRLK1L(-L). These preliminary findings pushed us to focus on the LRR XII(-L) group by running a window-based $\mathrm{Ka} / \mathrm{Ks}$ analysis in three $\mathrm{Pac}$ sequence sub-groups separately (clades I, II and IV + V,
Figures 2 and 7). The analysis showed an acceleration toward $\mathrm{Ka} / \mathrm{Ks}$ values higher than 1 in the first, central and final portions of the sequences, which corresponded, in the three groups, to the same sub-domains (Figure 7, Additional file 19). With regard to the average values of $\mathrm{Ka} / \mathrm{Ks}$ ratio per window, none were significantly larger than 1 (at pvalue $<0.05$ ). This is in line with a complete relaxation of purifying selection. In the LRR XII of Arabidopsis, the analysis was performed in two groups of sequences, one of which comprised AT4G08850 and AT1G35710 which did not align with the others according to a codon-delimited fashion. In Arabidopsis average $\mathrm{Ka} / \mathrm{Ks}$ values ranged from 0.01 to 0.34 , highlighting a signature of strong purifying selection. However the pattern of average $\mathrm{Ka} / \mathrm{Ks}$ values was similar to that observed in Pac (Figure 7, Additional file 19). In CrRLK1L-L, the average values ranged from 0.03 to 0.16 , thus showing the strongest signature of purifying selection (Figure 7, Additional file 19). In many windows of $\mathrm{Pac}$ sequences, $\mathrm{Ka} / \mathrm{Ks}$ values were significantly higher than those of the corresponding windows of Arabidopsis. Significant differences were also detected by comparing windows with the highest and the lowest values within each sequence group of $\mathrm{Pac}$ (see the table in Figure 7), in line with a differential action of the selection in different regions of the kinase domain. With regard to this type of comparison, a significant difference was also detected in Arabidopsis (see the table in Figure 7), (Wilcoxon-test pvalue $<0.0001$ and fold increase $>2$ ).

In the site by site analysis, the likelihood ratio test did not indicate an overall significantly better fit of the M8 site model (positive selection) versus the M7 model $(\mathrm{P}>0.05)$. The Bayes empirical Bayes method revealed a total of six sites with $\mathrm{Ka} / \mathrm{Ks}$ ratio $>1$, in the three sequence groups of $\mathrm{Pac}$, although with small posterior probability values (range 56-73\%) when accounting for the standard error.

A sequence analysis of each subgroup of LRR XII(-L) and of Arabidopsis LRR XII showed that of the 158 positions analysed (excluding gaps), 126 positions were subject to non-synonymous variations, of which $88(70 \%)$ were shared among two or more sequence groups, and $19(24 \%)$ were present in all the sequence groups (Figure 8). The amino acids recurring in all the shared positions and for each group of sequences are listed in Additional file 20. 


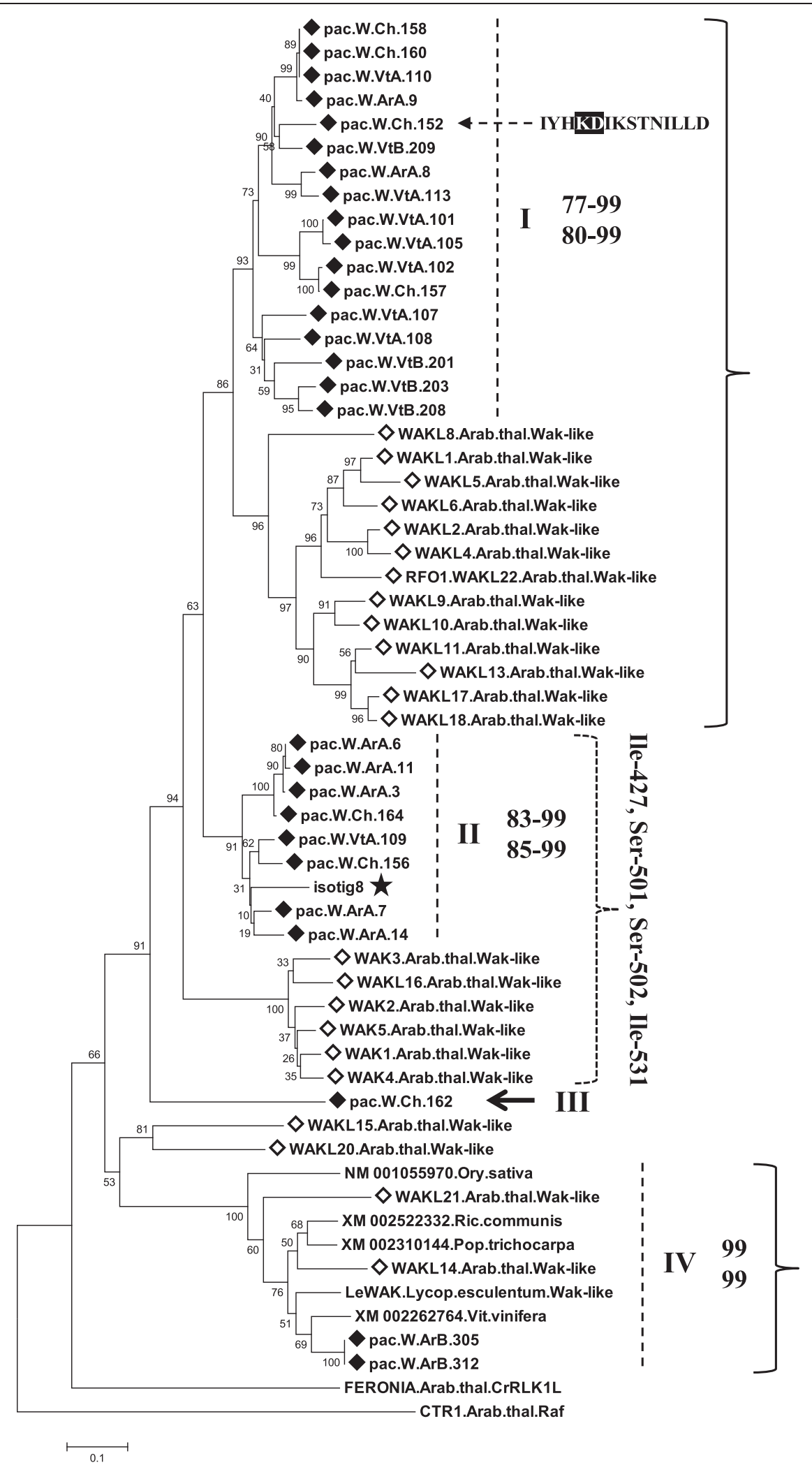

Figure 3 (See legend on next page.) 
(See figure on previous page.)

Figure 3 Neighbor-joining tree of Pac WAK-like candidates and Arabidopsis RLK/Pelle subfamily Wak-like. Analysis was based on the aa sequences of: Pac (pac ) obtained with Wak-like primers; the complete Wak-like subfamily of Arabidopsis ( $)$ ), other sequences from plant species and one isotig extracted from a 454 transcriptome dataset of Pac, which has the typical Wak-like extracellular domains (black star) (Additional file 5). The Pac sequence containing the non-RD motif is evidenced by a dashed arrow and the motif is shaded black. The kinase domain region spanned subdomain II up to the beginning of IX. The brackets indicate a likely genetic correspondence between Pac and Arabidopsis clades (uncertain in the case of the dotted bracket). Residues next to the brackets of clade II are shared by all the members of clade II and of WAK1-headed clade of Arabidopsis and differentiate these sequences from all the other Arabidopsis and Pac WAK-like (-L) (see Additional file 9). See caption of Figure 1 for additional details on the representation of the tree.

\section{Discussion}

Genome-wide analysis for the characterization of gene families in fully-sequenced species is invaluable for an in-depth comprehension of the information contained in the genomes (see for example: $[4,65,66])$. It is also a necessary support for gene candidate analyses such as those based on domain-targeted isolations of gene groups, in species lacking genome sequencing. We thus based our study on a detailed comparison with RLK/Pelles of fully-sequenced species, especially Arabidopsis, and devised a method for the correct identification of RLK/Pelle gene fragments.

In this study we present some substantial advances on plant RLK/Pelles. We isolated for the first time a set of RLK/Pelles in Pac. Interestingly, the analysis of sequence

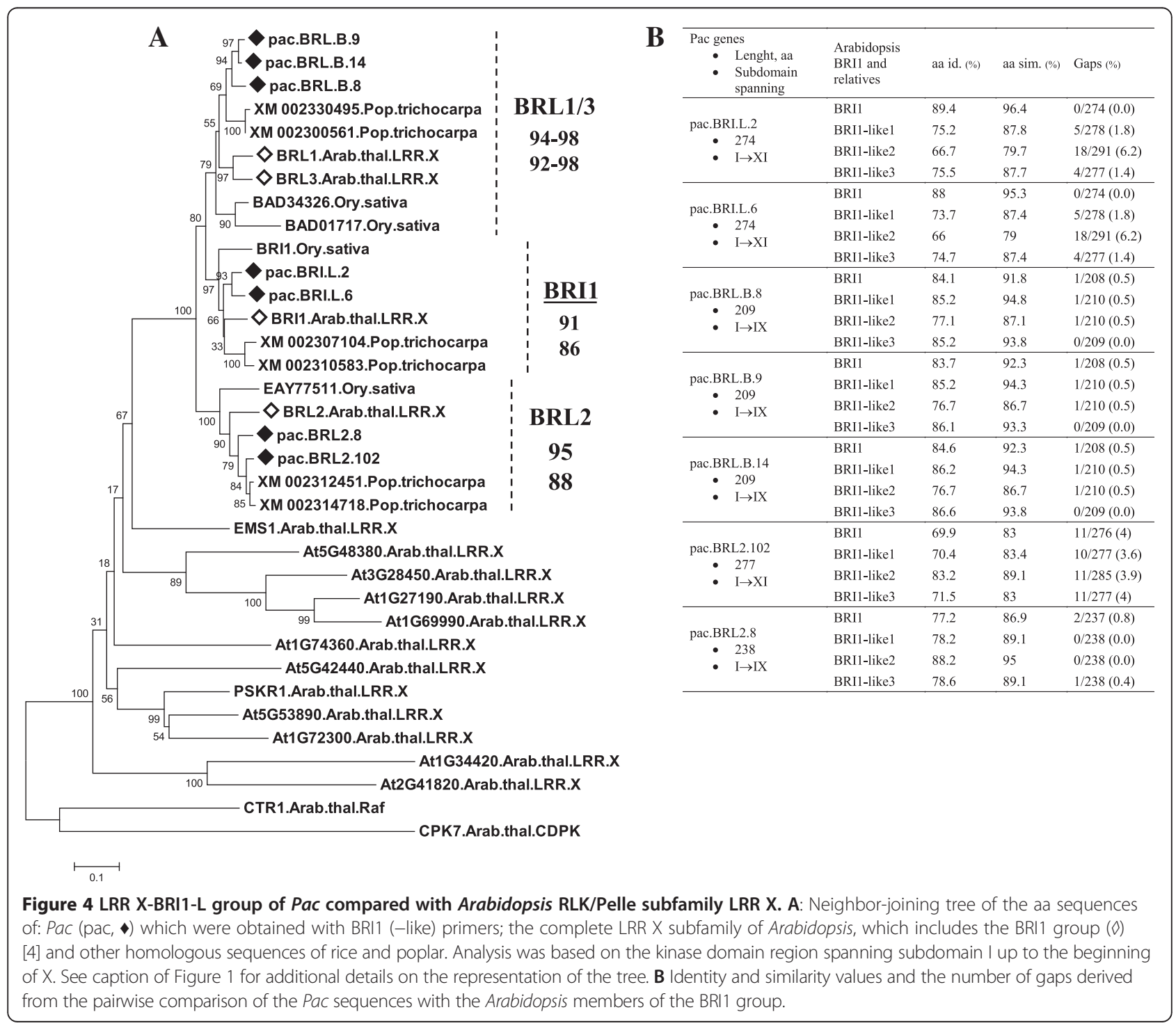




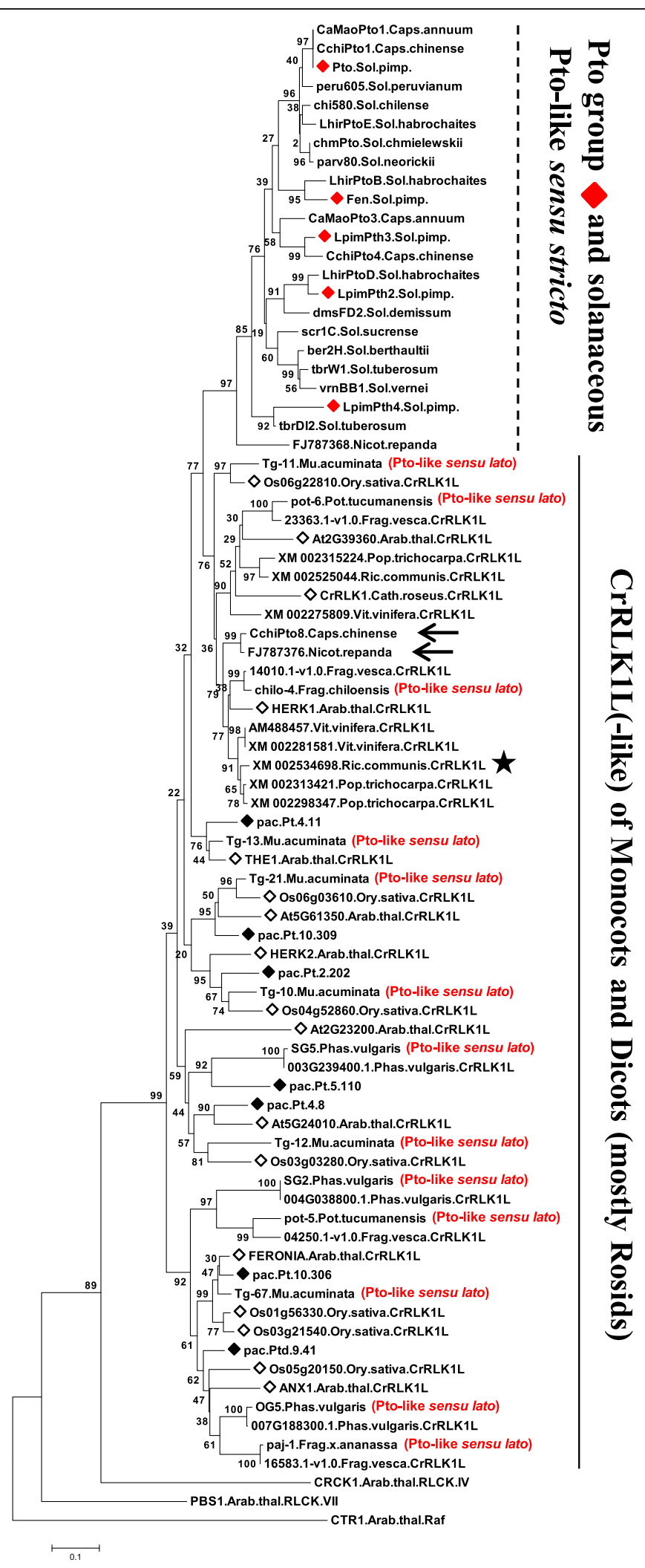

Figure 5 (See legend on next page.) 
(See figure on previous page.)

Figure 5 Phylogenetic relationship between Pto(-like) (sensu strictu and sensu latu) and the RLK/Pelle subfamily CrRLK1L. The Neighbor-joining analysis was based on the aa sequences of: Pto and paralogs of Solanum pimpinellifolium and the other solanaceous Pto-like (i.e. Pto-like sensu strictu); representative CrRLK1L of Arabidopsis $(\diamond)$, rice $(\diamond)$ and Catharanthus roseus ( () ); Pto best blastp matches (namely CrRLK1L, based on domain composition) of Arabidopsis, rice, grapevine, poplar and castor bean; Pto-like fragments of non-solanaceous species (Phaselus vulgaris, cultivated and wild strawberry and Musa acuminata) (namely CrRLK1L-like, i.e. Pto-like sensu latu); best blastp matches from F. vesca and P. vulgaris (namely CrRLK1L) obtained using Pto-like fragments from the same species, as query. Representative CrRLK1L-like of Pac obtained with Pto-primer were also included (pac, $\$$ ). Black arrows indicate the only solanaceous so-called Pto-like sequences, which clustered within a CrRLK1L-headed clade. The black star indicates a CrRLK1L of castor bean lacking the extracellular domain typical of the subfamily. The kinase domain region spanned subdomain I up to the beginning of IX. See caption of Figure 1 for additional details on the representation of the tree.

data also revealed: i) a novel phylogenetic relationship between the Pto group and the CrRLK1L RLK/Pelle subfamily; ii) clear signatures of relaxed purifying selection in the LRR XII-L, which had not been previously revealed for plant RLK kinase domain.

The representativeness of the kinase domain for RLK/ Pelle classification has been widely shown $[3-5,7,67,68]$. Kinase-domain targeted isolation of RLK/Pelles has also been found to be successful [69-75]. In this work we confirmed its representativeness for a correct classification, by matching the cloned sequences with long 454 isotigs from $\mathrm{Pac}$, which displayed the expected extracellular domain signatures (Additional file 5).

\section{CrRLK1L candidates and the relationship between CrRLK1L and Pto}

All the analyses highlighted our apparent failure to collect sensu strictu Pto-like genes in Pac and showed that the analysed non-solanaceous plant genomes lack a bona fide Pto gene group. Interestingly, in the phylogenetic analyses, Pto group was closest to CrRLK1L than any other RLK subfamily. Note that i) best matches of Pto, belonging to six fully-sequenced dicot species and to rice, were revealed as CrRLK1L; ii) all non-solanaceous Pto-like partial sequences were classified as CrRLK1L-like; in this regard it is explicative the high closeness of Pto-like partial sequences from bean and cultivated/wild strawberries to CrRLK1L extracted from bean and $F$. vesca genomes (Figure 5, Additional file 11). All the dicot species that we used in the Pto/CrRLK1L comparative analysis were Rosids, except the solanaceous species which belong to Asterids, and Pac, whose formation predates the split giving rise to Rosids and Asterids (http://www. mobot.org/MOBOT/research/APweb/). All this clearly points to the restriction of Pto gene group to a range of taxa within Dicots, possibly due to a relatively more recent origin than other ubiquitous RLK/Pelle subfamilies. On the other hand a more precise evaluation of the taxonomic restriction of Pto-like genes is needed and would require sequencing additional plant taxa representative of Asterids. The taxonomic restriction of an RLK group is not surprising as species-specific RLK/Pelle subfamilies have previously been reported [5,7].
The CrRLK1L subfamily is an ancient group of RLK/ Pelles with a conserved extracellular domain and whose formation predates the divergence of vascular plants $[5,50]$. This was confirmed by our phylogenies which clearly showed that orthologs are more similar than paralogs, and suggests that events giving rise to family members occurred prior to the monocot-dicot split and after the divergence rate was low (Figures 1 and 5, Additional files 7 and 12).

Strong evidence thus suggests that Pto genes evolved from ancient CrRLK1L, through the loss of the extracellular domain: i) the ubiquity of the CrRLK1L in the land plant and the conservation of this family over a long geological period [5,50]; ii) the restriction of Pto and its paralogs to asterid species (i.e. Solanaceae, based on current knowledge) (Figure 5, Additional files 11 and 12); iii) the close vicinity between the kinase domain of the two RLK/ Pelle groups (Figures 1 and 5, Additional files 6 and 12) [6]. Interestingly, the structure of the Pto group is also similar to CrRLK1L in that orthologs are more similar than paralogs [76]. Recently the tomato RLK/Pelle family has been characterised and the phylogenetic comparison with Arabidopsis showed that a CrRLK1L subfamily exists in tomato [6]. Interestingly we noted that Pto group was included in the CrRLK1L clade. Though this was not discussed by the authors it represents a full confirmation of the results of our phylogenies [6]. Phylogenetic vicinity between the kinase domains of RLK and RLCK subfamilies has been reported, and domain reassortment has been repeatedly emphasized as an ancient mechanism of RLK/Pelle evolution in the land plant lineage $[4,5,77]$. CrRLK1L is one of the Arabidopsis RLK/Pelle subfamilies which has corresponding RLPs (receptor-like proteins, i.e. proteins resembling the extracellular domain of RLKs), suggesting that these RLKs and their RLPs gave rise or were each derived from their counterpart [4]. In this scenario, our conclusion on the formation of RLCKs - Pto and paralogs - from RLKs - CrRLK1L - is thus in agreement with the accepted RLK/Pelle gene evolution model.

The vicinity between Pto group and CrRLK1L raises the question if they have to be classified as bona fide CrRLK1L or represent the founding members of a 
Table 2 Comparison of non-solanaceous Pto-like partial sequences with Pto and close relatives of CrRLK1L

\begin{tabular}{|c|c|c|c|c|}
\hline Pto-like genes & $\begin{array}{l}\text { Close phylogenetic relative } \\
\text { Pto }\end{array}$ & $\begin{array}{l}\text { aa id. } \\
\text { (\%) }\end{array}$ & $\begin{array}{l}\text { aa sim. } \\
\text { (\%) }\end{array}$ & Gaps (\%) \\
\hline \multirow{2}{*}{ chilo-4 Fragaria chiloensis } & At.HERK1 CrRLK1L & 91.3 & 95.6 & 0/183 (0.0) \\
\hline & Pto & 70.8 & 81.1 & $5 / 185(2.7)$ \\
\hline \multirow{2}{*}{ pot-6 Potentilla tucumanensis } & Cr.CrRLK1 & 80.6 & 91.9 & 3/186 (1.6) \\
\hline & Pto & 67.0 & 80.5 & $5 / 185(2.7)$ \\
\hline \multirow{2}{*}{ paj-1 Fragaria $\times$ ananassa } & At.ANX1 CrRLK1L & 80.4 & 91.3 & $1 / 184(0.5)$ \\
\hline & Pto & 63.4 & 79 & $6 / 186(3.2)$ \\
\hline \multirow{2}{*}{ Pot-5 Potentilla tucumanensis } & At.FERONIA CrRLK1L & 75.0 & 83.7 & $0 / 184(0.0)$ \\
\hline & Pto & 64.0 & 75.3 & $6 / 186(3.2)$ \\
\hline \multirow{2}{*}{ SG5 Phaseolus vulgaris } & At2G23200 CrRLK1L & 65.3 & 80.0 & $2 / 170(1.2)$ \\
\hline & Pto & 60.2 & 73.7 & $5 / 171(2.9)$ \\
\hline \multirow{2}{*}{ OG5 Phaseolus vulgaris } & At.ANX1 CrRLK1L & 84.3 & 91.3 & $2 / 172(1.2)$ \\
\hline & Pto & 63.4 & 78.5 & $3 / 172(1.7)$ \\
\hline \multirow{2}{*}{ SG2 Phaseolus vulgaris } & At.FERONIA CrRLK1L & 77.8 & 87.7 & 1/171 (0.6) \\
\hline & Pto & 60.7 & 75.7 & $7 / 173(4.0)$ \\
\hline \multirow{2}{*}{ Tg-10 Musa acuminata } & Os04g52860 CrRLK1L & 88.2 & 93.5 & $0 / 170(0.0)$ \\
\hline & Pto & 64.0 & 69.7 & $5 / 172(2.9)$ \\
\hline \multirow{2}{*}{ Tg-11 Musa acuminata } & Os06g22810 CrRLK1L & 91.2 & 95.3 & $0 / 170(0.0)$ \\
\hline & Pto & 68.6 & 80.8 & $5 / 172(2.9)$ \\
\hline \multirow{2}{*}{ Tg-12 Musa acuminata } & Os10g39010 CrRLK1L & 73.0 & 86.8 & $5 / 174(2.9)$ \\
\hline & Pto & 62.2 & 77.3 & $5 / 172(2.9)$ \\
\hline \multirow{2}{*}{ Tg-13 Musa acuminata } & At.THE1 CrRLK1L & 93.5 & 98.2 & $0 / 170(0.0)$ \\
\hline & Pto & 69.8 & 82.0 & $5 / 172(2.9)$ \\
\hline \multirow{2}{*}{ Tg-21 Musa acuminata } & Os06g03610 CrRLK1L & 92.9 & 96.5 & $0 / 170(0.0)$ \\
\hline & Pto & 65.5 & 79.5 & $5 / 171(2.9)$ \\
\hline \multirow{2}{*}{ Tg-67 Musa acuminata } & At.FERONIA CrRLK1L & 95.3 & 98.2 & 0/171 (0.0) \\
\hline & Pto & 61.8 & 75.7 & $6 / 173(3.5)$ \\
\hline \multirow{2}{*}{ pac.Pt.10.306 } & At.FERONIA CrRLK1L & 94.9 & 98.3 & $0 / 178(0.0)$ \\
\hline & Pto & 65.2 & 77.3 & $8 / 181(4.4)$ \\
\hline \multirow{2}{*}{ pac.Pt.10.309 } & At5G61350 CrRLK1L & 83.6 & 91.8 & 7/183 (3.8) \\
\hline & Pto & 68.5 & 81.5 & $5 / 178(2.8)$ \\
\hline \multirow{2}{*}{ pac.Ptd.9.41 } & At.ANX1 CrRLK1L & 83.1 & 93.8 & $0 / 177(0.0)$ \\
\hline & Pto & 68.2 & 80.4 & $5 / 179(2.8)$ \\
\hline \multirow{2}{*}{ pac.Pt.2.202 } & HERK2 CrRLK1L & 85.9 & 94.4 & $0 / 177(0.0)$ \\
\hline & Pto & 66.5 & 81.0 & $5 / 179(2.8)$ \\
\hline \multirow{2}{*}{ pac.Pt.4.11 } & At.THE1 CrRLK1L & 89.1 & 93.9 & $0 / 165(0.0)$ \\
\hline & Pto & 71.1 & 80.1 & $5 / 166(3.0)$ \\
\hline \multirow{2}{*}{ pac.Pt.4.8 } & At5G24010 CrRLK1L & 86.6 & 95.7 & $0 / 164(0.0)$ \\
\hline & Pto & 62.3 & 80.2 & $5 / 167(3.0)$ \\
\hline \multirow{2}{*}{ pac.Pt.5.110 } & At2G23200 CrRLK1L & 67.9 & 81.0 & $4 / 168(2.4)$ \\
\hline & Pto & 63.2 & 74.9 & 13/171 (7.6 \\
\hline
\end{tabular}

Pto primer-derived Pac sequences (pac) are included. At $=$ Arabidopsis thaliana; $\mathrm{Os}=$ Oryza sativa; $\mathrm{Cr}=$ Catharanthus roseus. 


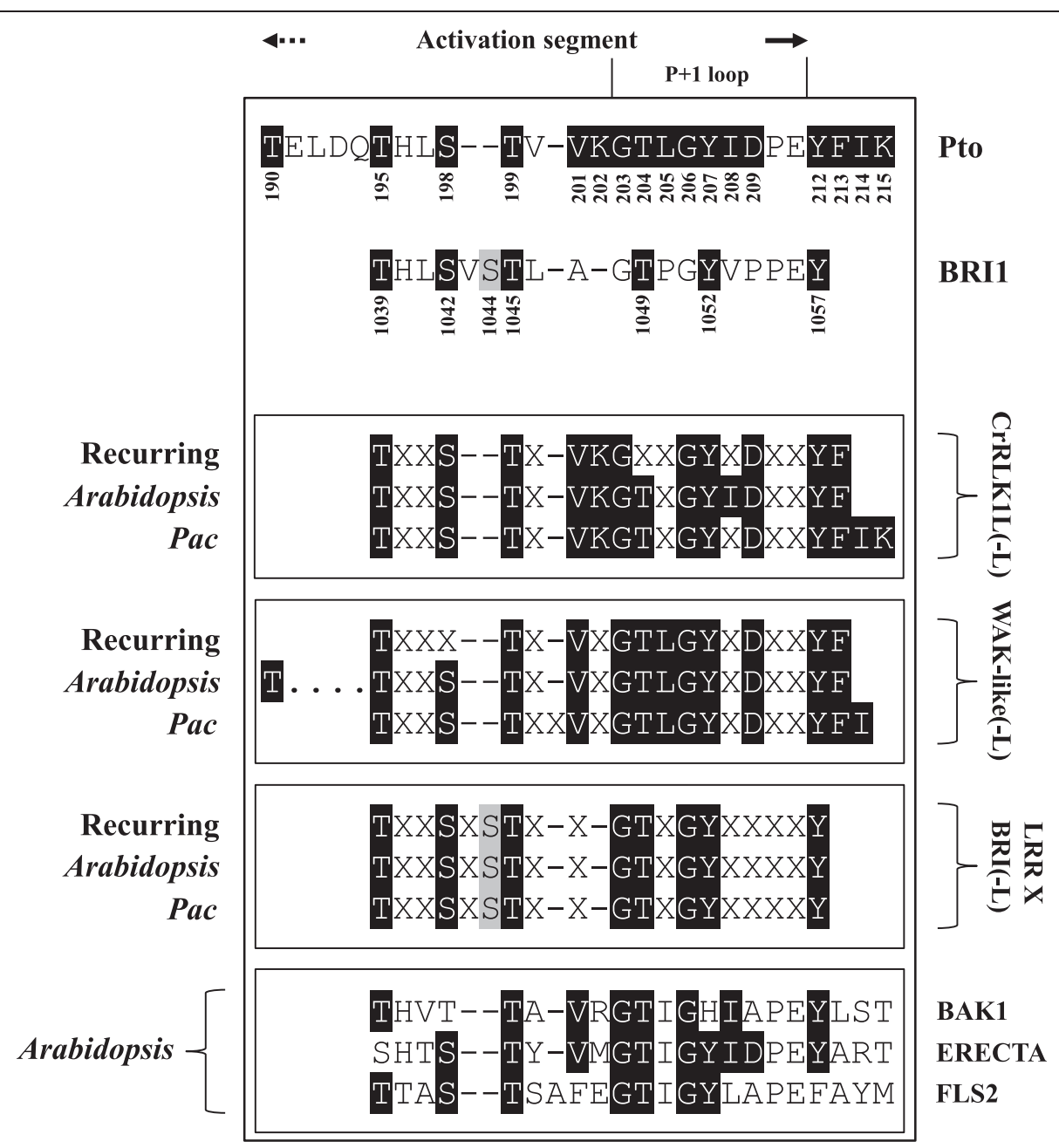

Figure 6 Functional consensus derived from Pto and BRI1 activation segments inferred from structure/function studies of other authors. These consensus motifs are compared with counterparts found in the activation segment of RLK/Pelle subfamilies of Arabidopsis and Pac separately. A consensus motif is also reported for each subfamily, which includes both species and only the most frequent residues of the Pto and BRI1 functional consensus (recurring). ERECTA, FLS2 and BAK1 are also included for comparison. Black-shaded residues define the Pto functional consensus and identities with the other RLK/Pelles. BRI1 consensus residues shared with Pto consensus motif, are shaded black. One residue was found to be exclusive of BRI1 and is shaded grey. In Arabidopsis and Pac RLK/Pelle subfamilies, residues that do not fall within the consensus positions are not reported $(X)$. The consensus motifs were derived from alignments presented in Additional files 16, 17 and 18, and manually refined.

novel RLCK/Pelle subfamily, related to CrRLK1L. This will be a matter of investigation and debate in the future.

The analysis of the activation segment was also useful for shedding light on the relationship between CrRLK1L(-like) and Pto (Figure 6). The functional motifs identified in the activation segment of BRI1 and Pto (Figure 6, Additional file 15), were partially overlapping and were conserved among a wide range of RLK/Pelles. This highlights an interesting point which fits the special case of CrRLK1L/ Pto well: the conservation of functional residues can be useful for supporting identification at a high hierarchical level but it is not sufficient to suggest a relationship of putative orthology, even when this conclusion is sustained by phylogenetic vicinity - as is the case of the non-solanaceous Pto-like vs solanaceous Pto group [73,74].

The recovery of a CrRLK1L $(-\mathrm{L})$ gene set in Pac, is still an interesting finding, also in the context of defense-related RLK/Pelles. For example THE1 mediates the response of plant cells to the perturbation of cellulose synthesis by triggering growth inhibition, ectopic lignin accumulation and the expression of a set of defense-related genes. THE1 is thus considered as a sensor of cell wall integrity and a role in pathogen defense has been postulated [78,79]. Another suggestion for the role of CrRLK1Ls in pathogen defense is that, in Arabidopsis, the bacterial effector AvrPto directly binds to the CrRLK1L member AT2G23200, suggesting a significant, yet undiscovered role, in innate immunity [63]. 


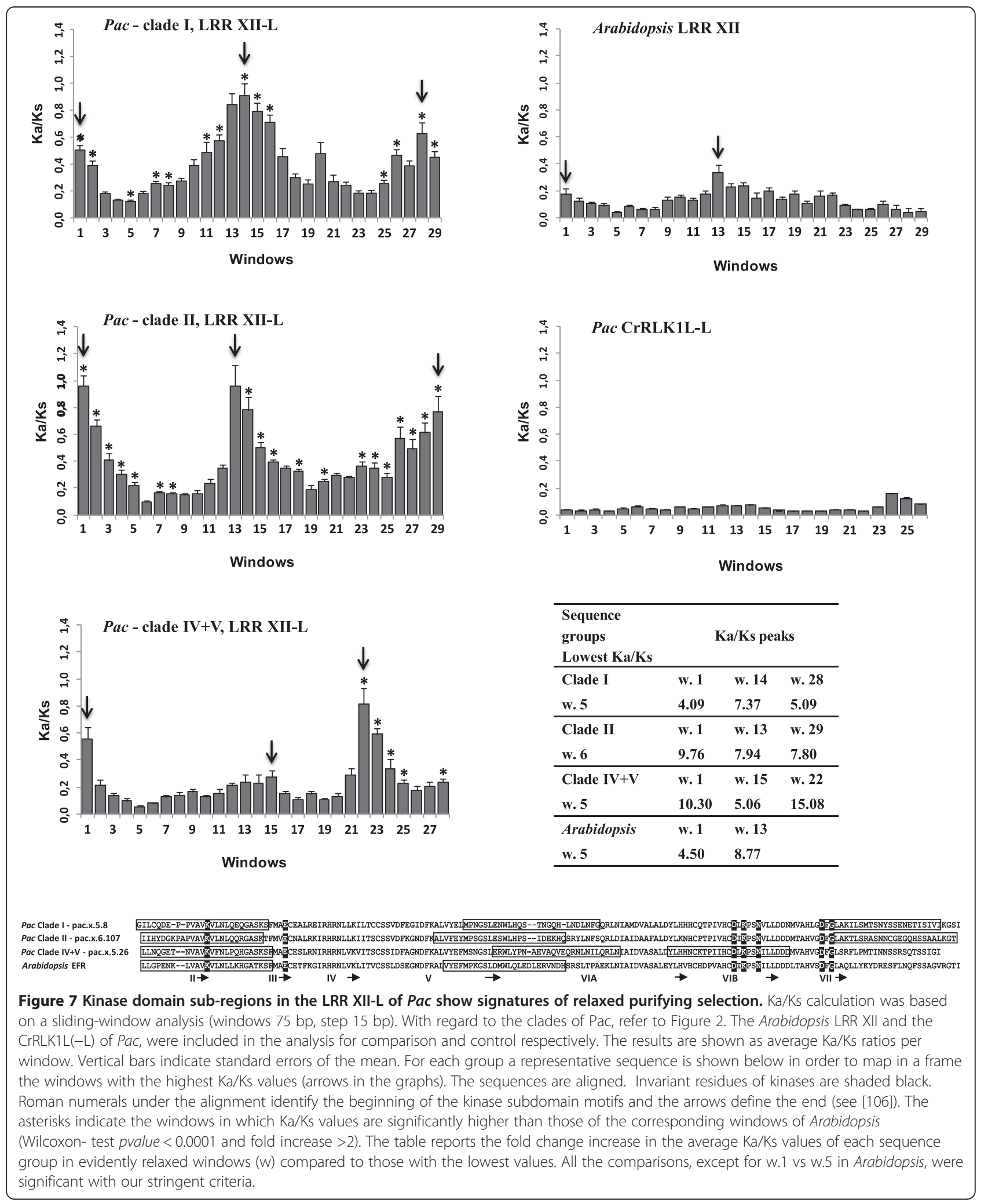




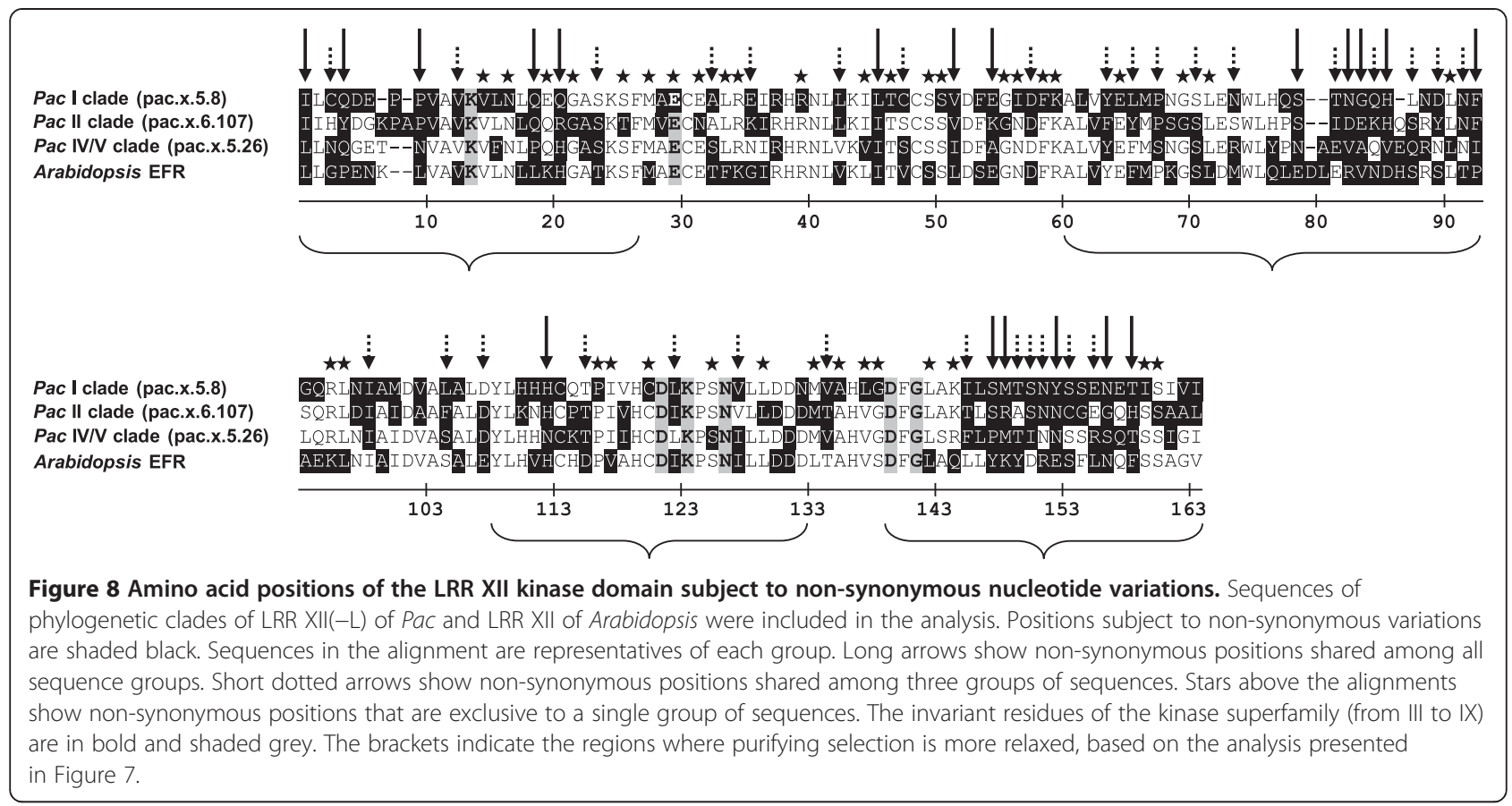

\section{LRR XII candidates: nonRD motif and relaxed purifying} selection highlight a potential link with pathogen resistance In Arabidopsis and rice the LRR XII subfamily includes PRRs functioning as PAMP receptors and $\mathrm{R}$ genes $[10,11,80]$ and it has been suggested that this is the general functional contribution of the subfamily. An LRR XII-L group was clearly identified in Pac (Figure 2, Additional file 6). The wide nucleotide diversity range among all $\mathrm{Pac}$ sequences (nucleotide score: 62-98) and a comparison, in the phylogenetic tree, of the branching pattern of Pac and Arabidopsis sequences, suggest that an undetermined number of paralogs had been isolated (Figure 2).

An important feature shared by the Arabidopsis and $\mathrm{Pac}$ sequence complements is their nature as non-RD kinases and the high frequency of Cys in substitution of the RD-Arg (Figure 2), which strongly supports the phylogenetic identification. Non-RD kinases are present in the kinomes of a wide range of Eukaryotic organisms and a statistically significant and positive correlation was found between kinases that function in the innate immunity and the non-RD motif [81]. This finding, together with the fact that Arabidopsis and rice LRR XII contain PRRs, suggests that we have identified a group of genes that is potentially involved in the perception of pathogens.

Further support for this hypothesis is that we also detected signatures of a relaxed purifying selection in $\mathrm{Pac}$ sequences (Figure 7). This finding is new for plant RLK/ Pelles. In fact in Arabidopsis and rice, signatures of positive selection were detected in the extracellular domains $[7,82,83]$. In contrast, the kinase domain has been shown to be under a strong purifying selection [7].
Interestingly, only specific regions of the kinase domains were subject, in $P a c$, to an evidently relaxed purifying selection and the substitutions occurring in the the non-synonymous positions were largely shared by the different sequence groups of Pac (Figures 7 and 8, Additional file 20). This suggests the action of a selective pressure that tends to keep specific non-silent variations in regions which might be relevant for the fitness. Indeed these regions span zones which are crucial for the function of the kinase catalytic core: i) I-II subdomain, which is involved in anchoring the ATP phosphates; ii) VII-VIII subdomain, the activation segment; iii) V-VIA subdomain, which is the site of catalysis $[84,85]$. The fact that the strength of purifying selection can be differentially modulated in the distinct functional regions of a protein coding gene is well known (e.g. [86,87]), however it is novel for plant RLK/Pelles.

Purifying selection generally eliminates any mutations in order to preserve the exact function of essential genes. On the other hand, a relaxation of purifying selection and positive selection are considered as two non-mutually exclusive forces that drive the protein evolutionary rate towards divergence. In particular in the relaxed purifying selection, random mutations affect genes that are dispensable or redundant, therefore there is no stringent constraint to impede their retention. By chance, these mutations may turn out to be beneficial for morphological and functional specification [88-90]. In fact a relaxed selective constraint plays a role in the expression of phenotypic plasticity, which is one of the most important ways by which organisms adaptively interact with the environment [90]. Specifically, 
the evolution of caste systems in polyphenic social insects [90], the fast evolution of lineage-specific genes in humans [91] and a variation in freezing tolerance among natural accessions of Arabidopsis [92] have all been attributed to a relaxed purifying selection as the main driving force.

It is well known that effectors of pathogenic bacteria target the kinase domain of several RLK/Pelles with a role in plant immunity, giving rise to an "arms race" ending with host resistance or susceptibility [20,62,63,93-96]. Thus in Pac, a relaxation of purifying selection in the kinase domain of LRR XII-L, may have enabled the recruitment of variants which are useful for the resistance response of $\mathrm{Pac}$ to some microbial (would be) pathogens. This might be an exciting research theme for the future.

\section{WAK-like and BRI1 group candidates}

WAK-like proteins link the extracellular matrix to the cytoplasm for appropriate signal transmission [97]. Wak-like have been found to be involved in many functions including pathogen resistance $[12,98,99]$ and development $[100,101]$. Despite our initial aim to isolate a single WAK-like member - namely an RFO1 homolog - a WAK-Like-L gene complement was isolated in Pac (Additional file 6) and seemed highly representative of the phylogenetic structure of the Arabidopsis counterpart (Figure 3). Importantly, results of WAK-like phylogeny agree exactly with the phylogenetic comparison between WAK-like of Arabidopsis and rice, in which sequences clustered in a species-specific manner and with only one multi-species clade headed by Arabidopsis WAKL14 and WAKL21 (Figure 3) [67]. However it remains difficult to predict the level of completeness of the gene complement isolated in Pac. In fact, compared with the Arabidopsis genome, an extensive expansion of the gene family has occurred both in rice and in poplar suggesting a lineageindependent evolution $[5,67]$. In any case the formation of species-specific and multi-species clades, in the Arabidopsis/Pac (this work) and Arabidopsis/rice phylogenies [67], suggests that opposing evolutionary forces shaped this RLK/Pelle subfamily, which produced both genetic divergence and conservation. This is in line with the involvement of these RLKs in very different functions, i.e. development and pathogen resistance, which exploit genetic conservation and diversification, respectively.

In the light of our results, as RFO1 belongs to a speciesspecific clade, phylogeny-based identification of the potential RFO1 homologs/orthologs was not possible. However based on the branching pattern of the phylogenetic tree, clade I appears to be the group of genes in which it might be possible to search for homologs with RFO1-related functions, i.e. resistance to vascular pathogens (Figure 3). This is particularly important in Platanus species which are hyper-susceptible to the vascular pathogen C. platani [26]. In Arabidopsis, a number of additional WAK-like are involved in stress responses, namely WAK1, WAK2, WAKL5 and WAKL7, which were found to be inducible by salicylic acid (SA) and by wounding, and to protect plants from detrimental effects during pathogen responses, such as high levels of SA $[98,100,101]$. In terms of finding orthologs of these WAK-like, clade II of Pac might be a candidate source, as it shares a 4-residue motif exclusively with the WAK1-headed clade, suggesting a genetic correlation (Additional file 9).

The topology of LRR X-BRI1-L phylogeny was the same as that found by Cano-Delgado et al. [102] based on full-length proteins of BRI1(-like) from plant species, which confirms once more that the kinase domain is of primary importance in RLK/Pelle subfamily identification and phylogeny. Similarly to CrRLK1(-L) phylogeny, the three major clades included the potential orthologs from different dicot and monocot species, implying that diversification within this gene group predates the monocot/ dicot split (Figure 4). Our analyses suggest that potential orthologs of BRI1 group members were isolated in Pac. In Arabidopsis, BRLs are expressed in the vasculature where they have a role in provascular cell growth and contribute to establishing the xylem/phloem pattern through perception of the BR signal [102,103]. In addition, BRLs also seem to be important in stress responses. In fact, after confirmation that BRI1 was not the functional receptor of systemin $[18,19]$ a BRL was suspected to be the true systemin receptor since BRLs match the highly specific localization of prosystemin and systemin perception in the vasculature $[104,105]$. Several Pac diseases target vascular tissues, cambium and living bark, and the pathogens gain entry through wounds [27]. Therefore, LRR X-BRI1-L of $\mathrm{Pac}$ are important candidates in the study of the host response to wound pathogens, such as C. platani.

\section{Conclusions}

The study of the RLK/Pelles of Pac led to interesting findings, highlighting some aspects of $P a c$ as a model species. This is also supported by the representative position of Platanus within Dicot phylogeny, one of the earliest branches, temporally located before the split which gave rise to the main groups, Rosids and Asterids, which comprise the majority of cultivated Dicots (http://www.mobot. org/MOBOT/research/APweb/.).

In addition the region-specific signatures of relaxed purifying selection in the non-RD LRR XII-L genes, highlights that this gene group is a good candidate for investigations into pathogen resistance.

With regard to CrRLK1L/Pto, it will be exciting to verify in which taxonomic groups, apart from solanaceae, sensu strictu Pto genes are currently present in order to further clarify the evolutionary link with CrRLK1L.

Above all, the main objective of our work in Pac was to create the first valuable resource to analyze the RLK kinome 
expression of genotypes that resist or are overwhelmed by pathogens. We believe that this represents the first step in an in-depth study on stress perception systems and also in the identification of useful molecular markers for genetic mapping and selecting resistant genotypes.

\section{Availability of supporting data}

The data set supporting the results of this article is available in the NCBI GenBank repository, (see also Additional file 3 for accession numbers and the text of nt and aa sequences).

\section{Additional files}

\section{Additional file 1: Degenerate primers successfully used for PCR amplifications of kinase domain of RLK/Pelle genes. \\ Additional file 2: Primer pairs used to isolate RLK/Pelle gene fragments and efficiency of the gene fishing process. \\ Additional file 3: Platanus $\times$ acerifolia $(P a c)$ RLK/Pelles: NCBI GenBank accession numbers, subfamily classification and sequence text. Sequences in $\mathbf{A}$ and $\mathbf{B}$ were obtained in this work, those in $\mathbf{C}$ were used for comparison. In $\mathbf{D}$ nucletide and amino acidic sequences are listed. \\ Additional file 4: Reference sequences included in the phylogenies for comparison and to root the trees. Where RLK subfamilies or generic terms (i.e. Pto-like) are cited, all sequences reported in the source were tested in the phylogenies, except pseudogenes or putative proteins with truncated kinase domains.}

Additional file 5: The extracellular domain of Platanus $\times$ acerifolia (Pac) RLK/Pelles. Pairwise comparison between selected gene fragments obtained in this work, and the overlapping RLK/Pelle isotigs extracted from a 454 FLX dataset of a Platanus $\times$ acerifolia (Pac) transcriptome (Pilotti M., Brunetti A., lacono M. and Pindo M. unpublished data). A Spanning regions and identity values. B The isotigs display a region 5'-upstream the kinase domain, which includes putative extracellular domains typical of the RLK/Pelle subfamilies dealt with in this work (output data from Pfam). This is a confirmation of the phylogenetic identification of the gene fragments. A SMART analysis predicted in a confident manner transmembrane domains in all the isotigs, which were located upstream the kinase domain and downstream the putative extracellular domains.

Additional file 6: Phylogenetic classification of RLK/Pelles of Platanus $\times$ acerifolia $(\mathrm{Pac})$. The analyses were based on the Neighbor-joining method and show a general view of the relationship of the aa sequences of RLK/Pelles of Pac (pac, in black), Pto group ( in red) and other kinases. A. Contains representative sequences of the following species/groups: Pac, Pto group (red rhombus), Pto-like partial sequences from non-solanaceous species (white rhombus with a red outline), RLK/Pelle subfamilies of Arabidopsis [4], other families of the receptor kinase group - receptor tyrosine kinase (RTK) and Raf [3] - and other eukaryotic protein kinases (ePKs). The tree is rooted with a bacterial protein kinase [APH( $\left.3^{\prime}\right)|l|$ also named aminoglycoside 3'-phosphotransferase]. B. Contains all Pac sequences reported in this work and their strict Arabidopsis homologs according to what emerged from $\mathbf{A}$. A kinase domain region spanning subdomains I/II to the beginning of VIII, was used to infer the trees. See caption of Figure 1 and Methods for details on the representation of the trees.

Additional file 7: The CrRLK1L-L of Platanus $\times$ acerifolia (Pac). Neighbor-joining analysis to compare CrRLK1L-L of Pac (pac, $\downarrow$ ), with the complete CrRLK1L subfamily of Arabidopsis and rice; Pto and paralogs of Solanum pimpinellifolium were also included (red rhombus); The subdivision into CRPK1-like 1 and CRPK1-like 2 is from Shiu and Bleecker [4]. aa sequences were used to infer the tree. Analysis was based on the kinase domain region spanning subdomain I through to IX. See caption of Figure 1 for details on the representation of the tree. Arrows indicate Arabidopsis CrRLK1L which were functionally characterised.
Additional file 8: Unwanted RLK/Pelles of Platanus $\times$ acerifolia (Pac). Sequences were obtained with primers aimed at isolating members of LRR XII subfamily. A Neighbor-joining tree based on the aa sequences of Pac (pac, $\bullet$ ), the nearest RLK/Pelle members of Arabidopsis $(\diamond)$ and other unknown, homologous sequences of rice and poplar. Analysis was based on the kinase domain region spanning subdomains $1 / \|$ to the beginning of VIII. See caption of Figure 1 for details on the representation of the tree. B Multiple alignment of RD motif (shaded grey), non-RD motif (shaded black) and an atypical variant of the non-RD-Asp (shaded black). C Phylogenetic analysis was ambiguous in determining the homology relationship of pac.Erf.2, pac.Erf.9 and pac.Erf.13 with the Arabidopsis RLK/Pelle subfamilies GDPD, LRK10L-2, CRPK1-like2 (i.e. CrRLK1L) and Thaumatin. The table thus shows the identity and similarity values and number of gaps derived from the pairwise comparison of these sequences with one Arabidopsis relative for each subfamily which, in preliminary analyses, were the nearest sequences.

Additional file 9: Wak1-clade-specific amino acid motif helps to identify close homologs in Platanus $\times$ acerifolia (Pac). Amino acidic residues (shaded black) conserved among sequences of Wak1-headed clade of Arabidopsis (RLK/Pelle subfamily WAK-like) and of the II phylogenetic clade of Wak-like- $L$ of Pac (refer to phylogeny of Figure 3 ). This motif suggests a genetic correlation between the two sequence groups.

Additional file 10: Classification of Pac sequences based on maximum-likelihood (ML) phylogenetic analyses. Analyses were performed on the same dataset alignments used for crucial Neighbor-joining trees presented in this work (aa sequences). A1 and A2 match with Additional file 6B (all Pac sequences compared with homologous Arabidopsis RLK Pelles); B matches with Additional file 7 (Arabidopsis and rice CrRLK1L vs Pac CrRLK1L-L); C matches with Figure 2 (Arabidopsis LRR XII vs Pac LRR XII-L); D matches with Figure 3 (Arabidopsis WAK-like vs Pac WAK-like-L); E with Figure 4 (Arabidopsis LRR X vs Pac LRR $X$-BRI1-L group). Editing matches that of counterpart figures. All trees were inferred with Mega 6 except the one represented in A2 that was TREE PUZZLE-implemented. With regard to Mega 6 analyses, the percentage of trees in which the associated sequences clustered together in the bootstrap test (1,000 replicates) is shown for each node; trees are drawn to scale in the number of substitutions per site (scale at the bottom). TREE-PUZZLE analysis was conducted with 50,000 puzzling steps and quartet puzzling support values are shown for each node.

Additional file 11: Phylogenetic relationship between Pto (-like) (sensu strictu and sensu latu) and the subfamily CrRLK1L by two methods of maximum-likelihood analysis. Analysis was performed on the same dataset alignment used for the analysis presented in Figure 5. The following aa sequences were included: Pto locus and solanaceous Pto-like; CrRLK1L of Arabidopsis, rice and Catharanthus roseus; Pto best blastp matches (namely CrRLK1L, based on domain composition) of Arabidopsis, rice, grapevine, poplar and castor bean; Pto-like partial sequences (namely CrRLK1L-like, i.e. Pto-like sensu latu) of non-solanaceous species (i.e. Phaselus vulgaris, cultivated and wild strawberry and Musa acuminata); best blastp matches from $F$. vesca and $P$. vulgaris (namely CrRLK1L) obtained using Pto-like partial sequences as query. Representative Pac sequences obtained with Pto-primer (CrRLK1L-like) were also included (pac). The arrows indicate solanaceous so-called Pto-like sequences, which clustered within a CrRLK1L-headed clade. The star indicates a CrRLK1L-like of castor bean lacking the extracellular domain typical of the subfamily. A: tree obtained with Mega 6; the percentage of trees in which the associated sequences clustered together in the bootstrap test (1,000 replicates) is shown for each node; trees are drawn to scale in the number of substitutions per site (scale at the bottom). B: tree obtained with TREE-PUZZLE; analysis was conducted with 50,000 puzzling steps and quartet puzzling support values are shown for each node. See Methods for details.

Additional file 12: Phylogenetic analyses (NJ and ML) to compare the complete CrRLK1L subfamily (CRPK1-like 1) of different plant species and Pto group. We included the complete CRPK1-like 1 aa sequence set of Arabidopsis, poplar, tomato (Solanum lycopersicum), rice and moss, and Pto and paralogs of Solanum pimpinellifolium (see Additional file 4 for sequence sources). The $\mathrm{NJ}$ tree is presented in $\mathbf{A}$, the ML trees, Mega6 and TREE-PUZZLE-implemented, are presented in $\mathbf{B}$ and $\mathbf{C}$ respectively. Analyses were based on the complete kinase domain region. With regard 
to Mega 6, the percentage of trees in which the associated sequences clustered together in the bootstrap test (1,000 replicates) is shown for each node; trees are drawn to scale in the number of substitutions per site (scale at the bottom). With regard to TREE-PUZZLE analysis was conducted with 50,000 puzzling steps and quartet puzzling support values are shown for each node. See Methods for additional details.

Additional file 13: The molecular role of some invariant residues of the kinase domain. Substitutions in Platanus $\times$ acerifolia (Pac) sequences (atypical and non-RD motifs) are listed.

Additional file 14: Serine, threonine and tyrosine phosphorilation specificity motifs of RLK/Pelles of Platanus $\times$ acerifolia (Pac) and Arabidopsis. The motifs are compared with phosphorilation specificity motifs of protein kinase previously inferred [42]. Consensus was determined using MEME (a motif-based sequence analysis tool).

Additional file 15: Crucial residues of the activation segments of Pto and BRI1. The relevant literature is cited in the text.

Additional file 16: Important residues of CrRLK1L located in the kinase domain region spanning subdomains VIB to VIII. The alignment shows the functional consensus derived from the Pto and BRI1 activation segment (subdomains VII and VIII) (Figure 6, Additional file 15) and its conservation in CrRLK1L(-L) subfamilies of Arabidopsis and Platanus $\times$ acerifolia (Pac). Black-shaded residues define the Pto functional consensus and the identities with CrRLK1L(-L). BRI1 consensus residues shared with Pto consensus motif, are shaded black, the residue exclusive of BRI1 consensus is shaded grey. Shaded-grey gaps have been inserted in order to match sequences with BRI1 consensus. The figure also shows the RD/non-RD motif and the regions that are useful to infer the ser/thr, tyr and the dual phosphorylation specificities of protein kinases [42] (Additional file 14) [red-shaded residues (subdomain Vlb) and the $\mathrm{P}+1$ loop (subdomain VIII)].

Additional file 17: Important residues of Wak-like located in the kinase domain region spanning subdomains VIB to VIII. The alignment shows the functional consensus derived from the Pto and BRI1 activation segment (subdomains VII and VIII) (Figure 6, Additional file 15) and its conservation in the WAK-like $(-\mathrm{L})$ subfamilies of Arabidopsis and Platanus $\times$ acerifolia (Pac). Black-shaded residues define the Pto functional consensus and the identities with WAK-like $(-L)$. BRI1 consensus residues shared with Pto consensus motif, are shaded black, the residue exclusive of BRl1 consensus is shaded grey. Shaded-grey gaps have been inserted in order to match sequences with BRI1 consensus. The figure also shows the RD and non-RD motif and the regions that are useful to infer the ser/thr, tyr and the dual phosphorylation specificities of protein kinases [42] (Additional file 14) [red-shaded residues (subdomain VIb) and the P + 1 loop (subdomain VIII)].

Additional file 18: Important residues of LRR X-BRI1 group located in the kinase domain region spanning subdomains VIB to VIII. The alignment shows the functional consensus derived from the Pto and BRI1 activation segment (subdomains VII and VIII) (Figure 6, Additional file 15) and its conservation in the LRR X-BRI1 (-L) group of Arabidopsis and Platanus $\times$ acerifolia (Pac). Black-shaded residues define the Pto functional consensus and the identities with LRR X-BRI1 (-L). BRI1 consensus residues shared with Pto consensus motif, are shaded black, the residue exclusive of BRI1 consensus is shaded grey. The figure also shows the RD motif and the regions that are useful to infer the ser/thr, tyr and the dual phosphorylation specificities of protein kinases [42] (Additional file 14) [red-shaded residues (subdomain Vlb) and the $\mathrm{P}+1$ loop (subdomain VIII)].

Additional file 19: $\mathrm{Ka} / \mathrm{Ks}$ values obtained for each window in the sliding window analysis of Platanus $\times$ acerifolia (Pac) LRR XII-L.

CrRLK1L-L of Pac and LRR XII of Arabidopsis are included for comparison.

Additional file 20: Amino acid variability at the kinase domain positions subject to non-synonymous variation. Analysis focuses on phylogenetic clades of LRR-XII-L of Platanus $\times$ acerifolia (Pac) and LRR-XII of Arabidopsis (refer Figure 2). The three distinct tables present the data regarding the sharing of the variable positions among four, three and two distinct group of sequences.

\section{Competing interests}

The authors declare that they have no competing interests.

\section{Authors' contributions}

The project was coordinated by MPil. MPil and AB designed the study and the gene isolation strategy. $A B, V L$ and $L T$ carried out nucleic acid extraction, PCR amplification and cloning. FG checked quality of Sanger-derived sequencing chromatograms and selected the RLK/Pelle sequences. MPil, PU, $\mathrm{Ml}$ and FG performed the bioinformatics and phylogenetic analyses. MPil, MI and MPin assembled the plane tree transcriptome and made the alignments to select the isotigs matching with PCR sequences of this work. MPil and $A B$ made figure editing. MPil prepared the manuscript. PU, AB, and VL critically reviewed the manuscript. All authors read and approved the final manuscript.

\section{Acknowledgements}

This research was supported by MIPAF_Ministero delle Politiche Agricole e Forestali, project: "Proteine e geni per la protezione delle piante dagli stress biotici e abiotici, Proteo Stress - Genomica e Proteomica per lo studio della risposta di resistenza in specie arbore, ciliegio e platano". We thank Adrian Wallwork for language editing.

\section{Author details}

${ }^{1}$ Plant Pathology Research Center, CRA-PAV Agricultural Research Council, V. C.G. Bertero 22, 00156 Rome, Italy. ${ }^{2}$ CRS4 Bioinformatics Laboratory POLARIS Science and Technology Park, 09010 Pula, Cagliari, Italy. ${ }^{3}$ Fruit Tree Research Center, CRA-FRU Agricultural Research Council, V. Fioranello, 52, 00134 Rome, Italy. ${ }^{4}$ Roche Diagnostics SpA, V. G.B. Stucchi 110, 20052 Monza, Milano, Italy. ${ }^{5}$ Research and Innovation Centre, Edmund Mach Foundation, V. E. Mach 1, 38010 San Michele a/A, Trento, Italy.

Received: 18 March 2014 Accepted: 18 November 2014 Published: 8 December 2014

\section{References}

1. Massagué J: TGF-beta signal transduction. Annu Rev Biochem 1998, 67:753-791.

2. Hubbard SR, Till JH: Protein tyrosine kinase structure and function. Annu Rev Biochem 2000, 69:373-398.

3. Shiu S-H, Bleecker AB: Receptor-like kinases from Arabidopsis form a monophyletic gene family related to animal receptor kinases. Proc Natl Acad Sci 2001, 98:10763-10768.

4. Shiu S-H, Bleecker AB: Expansion of the receptor-like kinase/pelle gene family and receptor-like proteins in Arabidopsis. Plant Physiol 2003, 132:530-543.

5. Lehti-Shiu MD, Zou C, Hanada K, Shiu S-H: Evolutionary history and stress regulation of plant receptor-like kinase/pelle genes. Plant Physiol 2009, 150:12-26.

6. Sakamoto T, Deguchi M, Brustolini OJB, Santos AA, Silva FF, Fontes EP: The tomato RLK superfamily: phylogeny and functional predictions about the role of the LRRII-RLK subfamily in antiviral defense. BMC Plant Biology 2012, 12:229.

7. Shiu SH, Karlowski WM, Pan R, Tzeng YH, Mayer KF, Li WH: Comparative analysis of the receptor-like kinase family in Arabidopsis and rice. Plant Cell 2004, 16:1220-1234.

8. Altenbach D, Robatzek S: Pattern-recognition receptors: from the cell surface to intracellular dynamics. Mol Plant Microbe Interact 2007, 20:1031-1039.

9. Zipfel C: Pattern-recognition receptors in plant innate immunity. Curr Op Immunol 2008, 20:10-16.

10. Park C-J, Han S-W, Chen X, Ronald PC: Elucidation of XA21-mediated innate immunity. Cell Microbiol 2010, 12:1017-1025.

11. Zipfel C, Kunze G, Chinchilla D, Caniard A, Jones JD, Boller T, Felix G: Perception of the bacterial PAMP EF-Tu by the receptor EFR restricts agrobacterium-mediated transformation. Cell 2006, 125:749-760.

12. Diener AC, Ausubel FM: Resistance to Fusarium oxisporum 1, a dominant Arabidopsis disease-resistance gene, is not race specific. Genetics 2005, 171:305-321.

13. Johansson A, Staal J, Dixelius C: Early responses in the Arabidopsis-Verticillium longisporum pathosystem are dependent on NDR1, JA- and ET-associated signals via cytosolic NPR1 and RFO1. Mol Plant Microbe Interact 2006, 19:958-969. 
14. He Z, Wang Z-Y, Li J, Zhu Q, Lamb C, Ronald P, Chory J: Perception of brassinosteroids by the extracellular domain of the receptor kinase BRI1. Science 2000, 288:2360-2363.

15. Wang Z-Y, Seto H, Fujioka S, Yoshida S, Chory J: BRI1 is a critical component of a plasma-membrane receptor for plant steroids. Nature 2001, 410:380-383.

16. Bajguz A, Hayat S: Effects of brassinosteroids on the plant responses to environmental stresses. Plant Phisiol Biochem 2009, 47:1-8.

17. Szekeres M: Brassinosteroid and systemin: two hormones perceived by the same receptor. Trends Plant Sci 2003, 8:102-104.

18. Holton N, Cano-Delgado A, Harrison K, Montoya T, Chory J, Bishop GJ: Tomato BRASSINOSTEROID INSENSITIVE1 is required for systemin-induced root elongation in solanum pimpinellifolium but is not essential for wound signaling. Plant Cell 2007, 19:1709-1717.

19. Malinowski R, Higgins R, Luo Y, Piper L, Nazir A, Bajwa VS, Clouse SD, Thompson PR, Stratmann JW: The tomato brassinosteroid receptor BRI1 increases binding of systemin to tobacco plasma membranes, but is not involved in systemin signaling. Plant Mol Biol 2009, 70:603-616.

20. Pedley KF, Martin GB: Molecular basis of PTO-mediated resistance to bacterial speck disease in tomato. Annu Rev Phytopathol 2003, 41:215-243.

21. Tang X, Xie M, Kim YJ, Zhou J, Klessig DF, Martin GB: Overexpression of Pto activates defense responses and confers broad resistance. Plant Cell 1999, 11:15-29.

22. Grimm GW, Denk T: The reticulate origin of modern plane trees (Platanus, Platanaceae): a nuclear marker puzzle. Taxon 2010, 59:134-147.

23. Henry A, Floods MG: The hystory of the London plane (platanus acerifolia). Notes on the genus Platanus. Proc Royal Irish Acad 1919, 35:9-28.

24. Pilotti M, Brunetti A, Tizzani L, Marani O: Platanus' acerifolia genotypes surviving to inoculation with Ceratocystis platani (the agent of canker stain): first screening and molecular characterization. Euphytica 2009, 169:1-17.

25. Soltis PS, Soltis DE: The role of genetic and genomic attributes in the success of polyploids. Proc Natl Acad Sci 2000, 97:7051-7057.

26. Panconesi $A$ : Canker stain of plane trees: a serious danger to urban plantings. J Plant Pathol 1999, 81:3-15.

27. Pilotti M: Le avversità del platano. Infre Fitopatol 2002, 52:9-24.

28. Vigouroux A, Olivier R: First hybrid plane trees to show resistance against canker stain (Ceratocystis fimbriata f. sp. platani). For Pathol 2004, 34:307-319.

29. Pilotti M, Brunetti A, Gallelli A, Loreti S: NPR1-like genes from cDNA of rosaceous trees: cloning strategy and genetic variation. Tree Genetics and Genomes 2008, 4:49-63.

30. Altschul SF, Madden TL, Schäffer AA, Zhang J, Zhang Z, Miller W, Lipman DJ: Gapped BLAST and PSI-BLAST: a new generation of protein database search programs. Nucleic Acids Res 1997, 25:3389-3402.

31. Katoh K, Toh H: Recent developments in the MAFFT multiple sequence alignment program. Brief Bioinform 2008, 4:286-298.

32. Rice $\mathrm{P}$, Longden I, Bleasby A: The European molecular biology open software suite. Trends Genet 2000, 16:276-277.

33. Saitou $N$, Nei M: The neighbor-joining method: a new method for reconstructing phylogenetic trees. Mol Biol Evol 1987, 4:406-425.

34. Zuckerkandl E, Pauling L: Evolutionary divergence and convergence in proteins. In Evolving Genes and Proteins. Edited by Bryson V, Vogel HJ. New York: Academic; 1965:97-166.

35. Tamura K, Stecher G, Peterson D, Filipski A, Kumar S: MEGA6: molecular evolutionary genetics analysis version 6.0. Mol Biol Evol 2013, 30:2725-2729.

36. Whelan S, Goldman N: A general empirical model of protein evolution derived from multiple protein families using a maximum likelihood approach. Mol Biol Evol 2001, 18:691-699.

37. Schmidt HA, Strimmer K, Vingron M, von Haeseler A: TREE-PUZZLE: maximum likelihood phylogenetic analysis using quartets and parallel computing. Bioinformatics 2002, 18:502-504.

38. Müller T, Vingron M: Modelling amino acid replacement. J Comput Biol 2000, 7:761-776.

39. Chang JH, Tai YS, Bernal AJ, Lavelle DT, Staskawicz BJ, Michelmore RW: Functional analyses of the Pto resistance gene family in tomato and the identification of a minor resistance determinant in a susceptible haplotype. Mol Plant Microbe Interact 2002, 15:281-291.

40. Finn RD, Mistry J, Tate J, Coggill P, Heger A, Pollington JE, Gavin OL, Gunesekaran P, Ceric G, Forslund K, Holm L, Sonnhammer EL, Eddy SR, Bateman A: The Pfam protein families database. Nucleic Acids Res 2010, 38:D211-D222. database issue.
41. Letunic I, Doerks T, Bork P: SMART 7: recent updates to the protein domain annotation resource. Nucleic Acids Res 2012, 40(D1):D302-D305.

42. Rudrabhatla P, Reddy MM, Rajasekharan R: Genome-wide analysis and experimentation of plant serine/threonine/tyrosine-specific protein kinases. Plant Mol Biol 2006, 60:293-319.

43. Bailey $T$, Elkan C: Fitting a mixture model by expectation maximization to discover motifs in biopolymers. In Proceedings of the Second International Conference on Intelligent Systems for Molecular Biology. Menlo Park, California: AAAI Press; 1994:28-36.

44. Steinway SN, Dannenfelser R, Laucius CD, Hayes JE, Nayak S: JCoDA: a tool for detecting evolutionary selection. BMC Bioinf 2010, 11:284.

45. Yang Z: PAML 4: phylogenetic analysis by maximum likelihood. Mol Biol Evol 2007, 24:1586-1591.

46. Yang Z, Nielsen R: Estimating synonymous and nonsynonymous substitution rates under realistic evolutionary models. Mol Biol Evol 2000, 17:32-43.

47. Felsenstein J: PHYLIP - phylogeny inference package (version 3.2). Cladistics 1989, 5:164-166.

48. Jones DT, Taylor WR, Thornton JM: The rapid generation of mutation data matrices from protein sequences. Comput Appl Biosci 1992, 8:275-282.

49. Verica JA, He Z-H: The cell wall-associated (WAK) and WAK-like kinase gene family. Plant Physiol 2002, 129:455-459.

50. Hématy K, Höfte H: Novel receptor kinases involved in growth regulation. Curr Opin Plant Biol 2008, 11:321-328.

51. Lejeune A, Constant S, Delavault P, Simier P, Thalouarn P, Thoiron S: Involvement of a putative Lycopersicon esculentum wall-associated kinase in the early steps of tomato-Orobanche ramosa interaction. Physiol Mol Plant Pathol 2006, 69:3-12.

52. Schallus T, Jaeckh C, Feher K, Palma AS, Liu Y, Simpson JC, Mackeen M, Stier G, Gibson TJ, Feizi T, Pieler T, Muhle-Goll C: Malectin: a novel carbohydrate-binding protein of the endoplasmic reticulum and a candidate player in the early steps of protein N-glycosylation. Mol Biol Cell 2008, 19:3404-3414.

53. Castells E, Casacuberta JM: Signalling through kinase-defective domains of atypical receptor-like kinases in plants. J Exp Bot 2007, 58:3503-3511.

54. Krupa A, Preethi G, Srinivasan N: Structural modes of stabilization of permissive phosphorilation sites in protein kinases: distinct strategies in Ser/Thr and Tyr kinases. J Mol Biol 2004, 339:1025-1039.

55. Huse M, Kuriyan J: The conformational plasticity of protein kinases. Cell 2002, 109:275-282.

56. Dong J, Xiao F, Fan F, Gu L, Cang H, Martin GB, Chai J: Crystal structure of the complex between Pseudomonas effector AvrPtoB and the tomato Pto kinase reveals both a shared and a unique interface compared with Avr-Pto. Plant Cell 2009, 21:1846-1859.

57. Wang X, Goshe MB, Soderblom EJ, Phinney BS, Kuchar JA, Li J, Asgami T, Yoshida S, Huber SC, Clouse SD: Identification and functional analysis of in vivo phosphorilation sites of the Arabidopsis BRASSINOSTEROID-INSENSITIVE1 receptor kinase. Plant Cell 2005, 17:1685-1703.

58. Scofield SR, Tobias CM, Rathjen JP, Chang JH, Lavelle DT, Michelmore RW, Staskawicz BJ: Molecular basis of gene for-gene specificity in bacterial speck disease of tomato. Science 1996, 274:2063-2065.

59. Frederick RD, Thilmony RL, Sessa G, Martin GB: Recognition specificity for the bacterial avirulence protein AvrPto is determined by Thr-204 in the activation loop of the tomato Pto kinase. Mol Cell 1998, 2:241-245.

60. Sessa G, D'Ascenzo M, Martin GB: Thr38 and Ser198 are Pto autophosphorilation sites required for the AvrPto-Pto-mediated hypersensitive response. Embo J 2000, 19:2257-2269.

61. Wu AJ, Andriotis VME, Durrant MC, Rathjen JP: A patch of surface-exposed residues mediates negative regulation of immune signalling by tomato Pto kinase. Plant Cell 2004, 16:2809-2821.

62. Xing W, Zou Y, Liu Q, Liu J, Luo X, Huang Q, Chen S, Zhu L, Bi R, Hao Q, Wu J-W, Zhou J-M, Chai J: The structural basis for activation of plant immunity by bacterial effector protein AvrPto. Nature 2007, 449:243-247.

63. Xiang T, Zong N, Zou Y, Wu Y, Zhang J, Xing W, Li Y, Tang X, Zhu L, Chai J, Zhou JM: Pseudomonas syringae effector AvrPto blocks innate immunity by targeting receptor kinases. Curr Biol 2008, 18:74-80.

64. Oh M-H, Wang X, Kota U, Goshe MB, Clouse SD, Huber SC: Tyrosine phosphorylation of the BRI1 receptor kinase emerges as a component of brassinosteroid signaling in Arabidopsis. Proc Natl Acad Sci 2009, 106:658-663.

65. Meyers B, Kozik A, Griego A, Kuang H, Michelmore RW: Genome-wide analysis of NBS-LRR-encoding genes in Arabidopsis. Plant Cell 2003, 15:809-834 
66. Kohler A, Rinaldi C, Duplessis S, Baucher M, Geelen D, Duchaussoy F, Meyers BC, Boerjan W, Martin F: Genome-wide identification of NBS resistance genes in Populus trichocarpa. Plant Mol Biol 2008, 66:619-636.

67. Zhang S, Chen C, Li L, Meng L, Singh J, Jiang N, Deng XW, He ZH, Lemaux PG: Evolutionary expansion, gene structure, and expression of the rice wall-associated kinase gene family. Plant Physiol 2005, 139:1107-1124.

68. Bouwmeester K, Govers F: Arabidopsis L-type lectin receptor kinases: phylogeny, classification, and expression profiles. J Exp Bot 2009, 60:4383-4396.

69. Vallad G, Rivkin M, Vallejos C, McClean P: Cloning and modelling of a Pto-like protein kinase family of common bean (Phaseolus vulgaris L.). Theor Appl Genet 2001, 103:1046-1058.

70. Vleeshouwers VGAA, Martens A, Van Dooijeweert W, Colon LT, Govers F, Kammoun S: Ancient diversification of the PTO kinase family preeceded speciation in Solanum. Mol Plant Microbe Interac 2001, 14:996-1005.

71. Deng Z, Gmitter FG: Cloning and characterization of receptor kinase class disease resistance gene candidates in Citrus. Theor Appl Genet 2003, 108:53-61.

72. Di Gaspero G, Cipriani G: Nucleotide binding site/leucine-rich repeats, Pto-like and receptor-like kinases related to disease resistance in grapevine. Mol Gen Genomics 2003, 269:612-623.

73. Peraza-Echeverria S, James-Kay A, Canto-Canchè B, Castillo-Castro E: Structural and phylogenetic analysis of Pto-type disease resistance gene candidates in banana. Mol Genet Genomics 2007, 278:443-453.

74. Martínez Zamora MG, Castagnaro AP, Díaz Ricci JC: Genetic diversity of Pto-like serine/threonine kinase disease resistance genes in cultivated and wild strawberries. J Mol Evol 2008, 67:211-221.

75. Gao Y, Xu Z, Jiao F, Yu H, Xiao B, Li Y, Lu X: Cloning, structural features, and expression analysis of resistance gene analogs in tobacco. Mol Biol Reports 2010, 37:345-354.

76. Rose LE, Michelmore RW, Langley $\mathrm{CH}$ : Natural variation in the Pto disease resistance gene within species of wild tomato (Lycopersicon). II. Population genetics of Pto. Genetics 2007, 175:1307-1319.

77. Sasaki G, Katoh K, Hirose N, Suga H, Kuma K, Miyata T, Su ZH: Multiple receptor-like kinase cDNAs from liverwort Marchantia polymorpha and two charophycean green algae, Closterium ehrenbergii and Nitella axillaris: Extensive gene duplications and gene shufflings in the early evolution of streptophytes. Gene 2007, 401:135-144.

78. Hématy K, Sado PE, Van Tuinen A, Rochange S, Desnos T, Balzergue S, Pelletie S, Renou JP, Hofte $\mathrm{H}$ : A receptor-like kinase mediates the response of Arabidopsis cells to the inhibition of cellulose synthesis. Curr Bio/ 2007, 17:922-931.

79. Postel S, Kemmerling B: Plant systems for recognition of pathogenassociated molecular patterns. Semin Cell Dev Biol 2009, 20:1025-1031.

80. Chinchilla D, Bauer Z, Regenass M, Boller T, Felix G: The Arabidopsis receptor kinase FLS2 binds flg22 and determines the specificity of flagellin perception. Plant Cell 2006, 18:465-476.

81. Dardick C, Ronald P: Plant and animal recognition receptors signal through non-RD kinases. Public Library of Science for Pathogens 2006, 2:14-28.

82. Strain E, Muse SV: Positively selected sites in the Arabidopsis receptor-like kinase gene family. J Mol Evol 2005, 61:325-332.

83. Sun $X$, Cao $Y$, Wang S: Point mutations with positive selection were a major force during the evolution of a receptor-kinase resistance gene family of rice. Plant Physiol 2006, 140:998-1008.

84. Hanks SK, Hunter T: Protein kinases 6. The eukaryotic protein kinase superfamily: kinase (catalytic) domain structure and classification. Faseb J 1995, 9:576-596

85. Johnson LN, Noble ME, Owen DJ: Active and inactive protein kinases: structural basis for regulation. Cell 1996, 85:149-158.

86. Aagaard JA, Willis JH, Phillips PC: Relaxed selection among duplicate floral regulatory genes in Lamiales. J Mol Evol 2006, 63:493-503.

87. Hileman L, Baum D: Why do paralogs persist? Molecular evolution of CYCLOIDEA and related floral symmetry genes in Antirrhineae (Veronicaceae). Mol Biol Evol 2003, 20:591-600.

88. Hahn MW: Distinguishing among evolutionary models for the maintenance of gene duplicates. J Heredity 2009, 100:605-617.

89. Innan $\mathrm{H}$, Kondrashov F: The evolution of gene duplications: classifying and distinguishing between models. Nat Rev Gen 2010, 11:97-108.
90. Hunt BG, Ometto L, Wurm Y, Shoemaker DW, Yi SV, Keller L, Goodisman MAD Relaxed selection is a precursor to the evolution of phenotypic plasticity. Proc Natl Acad Sci 2011, 108:15936-15941.

91. Cai JJ, Petrov DA: Relaxed purifying selection and possibly high rate of adaptation in primate lineage-specific genes. Genome Biol Evol 2010, 2:393-409.

92. Zhen $Y$, Ungerer MC: Relaxed selection on the CBF/DREB1 regulatory genes and reduced freezing tolerance in the Southern range of Arabidopsis thaliana. Mol Biol Evol 2008, 25:2547-2555.

93. Jones JDG, Dangl JL: The plant immune system. Nature 2006, 444:323-329.

94. Meng X, Bonasera JM, Kim JF, Nissinen RM, Beer SV: Apple proteins that interact with $\mathrm{DspA} / \mathrm{E}$, a pathogenicity effector of Erwinia amylovora, the fire blight pathogen. Mol Plant Microbe Interact 2006, 19:53-61.

95. Shan L, He P, Li J, Heese A, Peck SC, Nurnberger T, Martin GB, Sheen J: Bacterial effectors target the common signaling partner BAK1 to disrupt multiple MAMP receptor-signaling complexes and impede plant immunity. Cell Host Microbe 2008, 4:17-27.

96. Gimenez-Ibanez S, Hann DR, Ntoukakis V, Petutschnig E, Lipka V, Rathjen JP: AvrPtoB targets the LysM receptor kinase CERK1 to promote bacterial virulence on plants. Curr Biol 2009, 19:423-429.

97. Anderson CM, Wagner TA, Perret M, He ZH, He D, Kohorn BD: WAKs: cell wall-associated kinases linking the cytoplasm to the extracellular matrix. Plant Mol Biol 2001, 47:197-206.

98. He Z-H, He D, Kohorn BD: Requirement for the induced expression of a cell wall associated receptor kinase for survival during the pathogen response. Plant J 1998, 14:55-63.

99. Brutus A, Sicilia F, Macone A, Cervone F, De Lorenzo G: A domain swap approach reveals a role of the plant wall-associated kinase 1 (WAK1) as a receptor of oligogalacturonides. Proc Natl Acad Sci 2010, 107:9452-9457.

100. Wagner T, Kohorn B: Wall-associated kinases are expressed throughout plant development and are required for cell expansion. Plant Cell 2001, 13:303-318.

101. Verica JA, Chae L, Tong H, Ingmire P, He ZH: Tissue-specific and developmentally regulated expression of a cluster of tandemly arrayed cell wall-associated kinase-like kinase genes in Arabidopsis. Plant Physiol 2003, 133:1732-1746.

102. Cano-Delgado A, Yin Y, Yu C, Vafeados D, Mora-Garcia S, Cheng J-C, Nam KH, Li J, Chory J: BRL1 and BRL3 are novel brassinosteroid receptors that function in vascular differentiation in Arabidopsis. Development 2004, 131:5341-5351.

103. Clay NK, Nelson T: VH1, a provascular cell-specific receptor kinase that influences leaf cell patterns in Arabidopsis. Plant Cell 2001, 14:2707-2722.

104. Narvaez-Vasquez J, Ryan CA: The cellular localization of prosystemin: a functional role for phloem parenchyma in systemic wound signaling. Planta 2004, 218:360-369.

105. Hind SR, Malinowski R, Yalamanchili R, Stratmann JW: Tissue-type specific systemin perception and the elusive systemin receptor. Plant Signaling Behavior 2010, 5:42-44.

106. Diévart A, Clark SE: Using mutant alleles to determine the structure and function of leucine-rich repeat receptor-like kinases. Curr Op Plant Biol 2003, 6:507-516.

\section{doi:10.1186/1756-0500-7-884}

Cite this article as: Pilotti et al:: Kinase domain-targeted isolation of defense-related receptor-like kinases (RLK/Pelle) in Platanus $\times$ acerifolia: phylogenetic and structural analysis. BMC Research Notes 2014 7:884. 